Portland State University

PDXScholar

$1-1986$

\title{
Fock-Tani Transformation and a First-Order Theory of Charge Transfer
}

P. C. Ojha

University of Oregon

M. D. Girardeau

University of Oregon

J. D. Gilbert

University of Oregon

Jack C. Straton

Portland State University, straton@pdx.edu

Follow this and additional works at: https://pdxscholar.library.pdx.edu/phy_fac

Part of the Atomic, Molecular and Optical Physics Commons

Let us know how access to this document benefits you.

\section{Citation Details}

Ojha, P. C., M. D. Girardeau, J. D. Gilbert, and J. C. Straton. "Fock-Tani transformation and a first-order theory of charge transfer." Physical Review A 33, no. 1 (1986): 112. DOI: http://dx.doi.org/10.1103/ PhysRevA.33.112

This Article is brought to you for free and open access. It has been accepted for inclusion in Physics Faculty Publications and Presentations by an authorized administrator of PDXScholar. Please contact us if we can make this document more accessible: pdxscholar@pdx.edu. 


\title{
Fock-Tani transformation and a first-order theory of charge transfer
}

\author{
P. C. Ojha, M. D. Girardeau, J. D. Gilbert, and J. C. Straton \\ Department of Physics, Institute of Chemical Physics and Institute of Theoretical Science, \\ University of Oregon, Eugene, Oregon 97403 \\ (Received 28 May 1985)
}

\begin{abstract}
A unitary (Fock-Tani) transformation of the second-quantized Hamiltonian breaks the interaction into its component parts, e.g., elastic scattering, inelastic scattering, rearrangement interaction, etc. The interaction for a particular process is "weaker" than the overall interaction; this is reflected in certain orthogonality corrections which appear in a perturbation expansion of the $T$-matrix element. As a result, the internuclear potential makes a negligible contribution of order $m_{e} / m_{p}$ to the firstorder amplitude for charge transfer. We find very good agreement with experimental and the best available theoretical results for the total cross section for the reaction $p+\mathbf{H}(1 s) \rightarrow \mathbf{H}(1 s)+p$ for energies greater than $10 \mathrm{keV}$ and for the differential cross section at 25, 60, and $125 \mathrm{keV}$ in an angular range of $\sim 1 \mathrm{mrad}$ about the forward direction.
\end{abstract}

\section{INTRODUCTION}

Atomic physics provides the ideal setting for testing new approaches to the quantum-mechanical scattering problem mainly because the interaction potential is known but also because of the variety and richness of phenomena open to investigation. This paper is part of a broader program to investigate the suitability of one such approach based on unitary (Fock-Tani) transformation of the second-quantized Hamiltonian. The motivation for this transformation is discussed and its basic features illustrated for the simple case of potential scattering in Sec. II; the mathematical details relevant to present application are given in Sec. III and the complete result is enumerated in Appendix A. Here we merely note the final result that the interaction is broken into its component parts, e.g., elastic scattering, inelastic scattering, rearrangement interaction, etc. This gives one considerably more latitude in introducing physically motivated approximations, for example, in a Born series expansion of the scattering amplitude. The effective interaction for a particular process is, in a sense, "weaker" than the overall interaction which is the sum of all interactions. It is found that to each order in the perturbation, certain orthogonality corrections to the usual (Fock) scattering amplitude appear. In this paper we restrict ourselves to a first-order (Born-type) approximation, in which case the orthogonality correction becomes negligible at high energies. We therefore expect the Fock-Tani formalism to extend the range of validity of the first-order approximation to lower energies.

This formalism has been applied previously to the calculation of the scattering amplitude for positronium formation $^{1}$ and for positron-hydrogen elastic scattering ${ }^{2}$ in collisions of positrons with hydrogen atoms. The numerical results of the second of these papers clearly demonstrate the superiority of the Fock-Tani representation over close-coupling representations in some cases where channel coupling plays an important role. In this paper we consider the closely related problem of resonant charge transfer,

$$
p+\mathbf{H}(1 s) \rightarrow \mathbf{H}(1 s)+p
$$

with the understanding that at collision energies under consideration (relative velocities of order 1 a.u.) protonproton exchange plays no role. This problem, in spite of a long history of quantum-mechanical analysis going back to the early days of quantum mechanics, ${ }^{3,4}$ is still under vigorous investigation as indicated by a recent review arti$\mathrm{cle}^{5}$ and numerous other publications. An early difficulty was caused by the Coulomb repulsion of the nuclei. This was neglected by Oppenheimer ${ }^{3}$ and Brinkman and Kramers ${ }^{4}$ on physical grounds but the resulting cross sections were too large by a factor of 3 or 4 . Its inclusion in a mathematically consistent first-order theory 6,7 brought the total cross section in line with experiment but clashed with the physical argument that the internuclear potential cannot significantly affect the cross section for charge transfer. In Sec. IV we show that in the Fock-Tani formalism, as a consequence of the orthogonality correction, the Coulomb interaction of the nuclei contributes a negligible term of order $m_{e} / m_{p}$ to the first-order reaction amplitude, a result pleasingly consistent with an observation about the exact scattering amplitude originally made by Wick (see footnote in Ref. 6).

The first-order orthogonality correction is reduced to a numerically tractable form in Sec. V, and computed differential and total cross sections are compared with experiment and the best available theoretical results (two-state atomic expansion introduced by Bates ${ }^{8}$ ) in Sec. VI. In Sec. VII we summarize our results and outline possible future extension of this work.

\section{FOCK-TANI TRANSFORMATION FOR POTENTIAL SCATTERING}

The basic idea behind Fock-Tani transformation and its consequence for scattering are best explained for scattering of a single particle from a fixed potential $V(\mathbf{r})$ which is sufficiently strong to support a number of bound states $\{|\mu\rangle, \mu=1,2,3, \ldots\}$ with energies $\left\{\epsilon_{\mu}, \mu=1,2,3, \ldots\right\}$. 
This formulation was first considered by $\operatorname{Tani}^{9}$ but the following summary is closer in its mathematical detail to the generalization due to Girardeau. ${ }^{10}$

We begin with the second-quantized form of the Hamiltonian (Fock Hamiltonian)

$$
\hat{H}_{F}=\int d \mathbf{r} \hat{a}^{\dagger}(\mathbf{r})[T(\mathbf{r})+V(\mathbf{r})] \hat{a}(\mathbf{r}),
$$

where $T(\mathbf{r})$ is the kinetic-energy operator and $\hat{a}^{\dagger}(\mathbf{r})$ and $\hat{a}(\mathbf{r})$ are "elementary" creation and annihilation operators defined in the Fock space $\mathscr{F}$. These satisfy canonical commutation relations

$$
\left[\hat{a}(\mathbf{r}), \hat{a}\left(\mathbf{r}^{\prime}\right)\right]=\left[\hat{a}^{\dagger}(\mathbf{r}), \hat{a}^{\dagger}\left(\mathbf{r}^{\prime}\right)\right]=0
$$

and

$$
\left[\hat{a}(\mathbf{r}), \hat{a}^{\dagger}\left(\mathbf{r}^{\prime}\right)\right]=\delta\left(\mathbf{r}-\mathbf{r}^{\prime}\right) .
$$

If the wave functions $\left\{\psi_{\mu}(\mathbf{r}), \mu=1,2, \ldots\right\}$ of the bound states are known, one may introduce "bound-state" creation and annihilation operators

$$
\hat{\psi}_{\mu}^{\dagger}=\int d \mathbf{r} \psi_{\mu}(\mathbf{r}) \hat{a}^{\dagger}(\mathbf{r})
$$

and

$$
\hat{\psi}_{\mu}=\int d \mathbf{r} \psi_{\mu}^{*}(\mathbf{r}) \hat{a}(\mathbf{r}) .
$$

Note that the commutation relations of these operators with $\hat{a}(\mathbf{r})$ and $\hat{a}^{\dagger}(\mathbf{r})$ are not always simple. In particular,

$$
\left[\hat{a}(\mathbf{r}), \hat{\psi}_{\mu}^{\dagger}\right]=\psi_{\mu}(\mathbf{r})
$$

and

$$
\left[\hat{a}^{\dagger}(\mathbf{r}), \hat{\psi}_{\mu}\right]=-\psi_{\mu}^{*}(\mathbf{r})
$$

are noncanonical. This complicates the mathematical analysis whenever one has to make explicit the existence of bound states of the system.

It is therefore desirable to introduce operators which can be associated with the bound states and which satisfy canonical commutation relations instead of (2.4). With this in mind, we introduce an "ideal-state" space $\mathscr{B}$ in which creation and annihilation operators $\left\{\hat{b}_{\mu}^{\dagger}, \hat{b}_{\mu}\right.$, $\mu=1,2, \ldots\}$ are defined. These are further assumed to satisfy the following commutation relations:

$$
\left[\hat{b}_{\mu}, \hat{b}_{v}\right]=\left[\hat{b}_{\mu}^{\dagger}, \hat{b}_{v}^{\dagger}\right]=0
$$

and

$$
\left[\hat{b}_{\mu}, \hat{b}_{v}^{\dagger}\right]=\delta_{\mu v} .
$$

We next construct the direct-product space $\mathscr{H} \equiv \mathscr{F} \otimes \mathscr{B}$. In this space all annihilation and creation operators previously introduced are defined. However, the "ideal-state" operators $\hat{b}_{\mu}, \hat{b}_{\mu}^{\dagger}$, etc., unlike the "realstate" operators $\hat{\psi}_{\mu}, \hat{\psi}_{\mu}^{\dagger}$, etc., commute with the elementary operators $\hat{a}(\mathbf{r})$ and $\hat{a}^{\dagger}(\mathbf{r})$ :

$$
\begin{aligned}
{\left[\hat{a}(\mathbf{r}), \hat{b}_{\mu}\right] } & =\left[\hat{a}(\mathbf{r}), \hat{b}_{\mu}^{\dagger}\right] \\
& =\left[\hat{a}^{\dagger}(\mathbf{r}), \hat{b}_{\mu}\right]=\left[\hat{a}^{\dagger}(\mathbf{r}), \hat{b}_{\mu}^{\dagger}\right]=0 .
\end{aligned}
$$

The subspace $\mathscr{H}_{F} \subset \mathscr{H}$ defined by the condition

$$
\sum_{\mu} \hat{b}_{\mu}^{\dagger} \hat{b}_{\mu}|\chi\rangle=0 \text { iff }|\chi\rangle \in \mathscr{H}_{F}
$$

is quite obviously isomorphic to the Fock space $\mathscr{F}$. Any calculations in $\mathscr{F}$ may as well be done in $\mathscr{H}_{F}$. We will, however, make a unitary transformation which will give the physical meaning of the real states $\hat{\psi}_{\mu}^{\dagger}|0\rangle$ to the ideal states $\hat{b}_{\mu}^{\dagger}|0\rangle$. This transformation will carry the subspace $\mathscr{H}_{F} \subset \mathscr{H}$ to the Fock-Tani subspace $\mathscr{H}_{\mathrm{FT}} \subset \mathscr{H}$ in which all calculation with the transformed Hamiltonian should be done. This transformation, schematically indicated in Fig. 1, is defined via the unitary operator

$$
\hat{U}=\exp \left(\frac{\pi}{2} \sum_{\mu}\left(\hat{\psi}_{\mu}^{\dagger} \hat{b}_{\mu}-\hat{b}_{\mu}^{\dagger} \hat{\psi}_{\mu}\right)\right) .
$$

The transform $\hat{U}^{-1} \hat{O} \hat{U}$ of any operator is evaluated using the Campbell-Hausdorff formula. It is easily verified that the transformed Hamiltonian (Fock-Tani Hamiltonian) is given by

$$
\begin{aligned}
\mathscr{H}_{\mathrm{FT}} \equiv & \hat{U}^{-1} \mathscr{H}_{F} \hat{U} \\
= & \sum_{\mu} \epsilon_{\mu} \hat{b}_{\mu}^{\dagger} \hat{b}_{\mu}+\int d \mathbf{r} \hat{a}^{\dagger}(\mathbf{r})[T((\mathbf{r})+V(\mathbf{r})] \widehat{a}(\mathbf{r}) \\
& -\sum_{\mu} \epsilon_{\mu} \int d \mathbf{r} d \mathbf{r}^{\prime} \hat{a}^{\dagger}(\mathbf{r}) \psi_{\mu}(\mathbf{r}) \psi_{\mu}^{*}\left(\mathbf{r}^{\prime}\right) \hat{a}\left(\mathbf{r}^{\prime}\right),
\end{aligned}
$$

and that

$$
\widehat{U}^{-1} \hat{b}_{\mu} \hat{U}=-\hat{\psi}_{\mu}
$$

and

$$
\widehat{U}^{-1} \widehat{\psi}_{\mu} \hat{U}=\hat{b}_{\mu} .
$$

From (2.10) it is clear that the role of $\hat{b}_{\mu}$ and $\hat{\psi}_{\mu}$ is interchanged. In fact, a weaker condition [which holds in the general case of scattering from targets with internal structure even when (2.10) does not]

$$
\hat{\psi}_{\mu}^{\dagger}|0\rangle \rightarrow \hat{b}_{\mu}^{\dagger}|0\rangle
$$

i.e., a real state $\hat{\psi}_{\mu}^{\dagger}|0\rangle$ is transformed into an ideal state
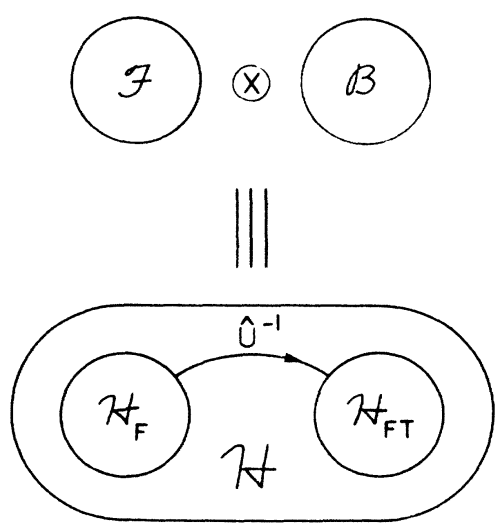

FIG. 1. Schematic connection between various Hilbert spaces defined in the text. Fock-Tani transformation is effected by the operator $\hat{U}^{-1}$ which carries $\mathscr{H}_{F}$ into $\mathscr{H}_{\text {FT }}$. 
$\hat{b}_{\mu}^{\dagger}|0\rangle$, is sufficient proof that the transformation gives physical meaning to the ideal states.

The subsidiary condition (2.7) transforms to

$$
\sum_{\mu} \hat{\psi}_{\mu}^{\dagger} \hat{\psi}_{\mu}|\chi\rangle=0 \text { iff }|\chi\rangle \in \mathscr{H}_{\text {FT }} \text {. }
$$

Equation (2.9) may be viewed as a formal expression of the following operator identity:

$$
\hat{H} \equiv \sum_{\mu} \epsilon_{\mu} P_{\mu}+(1-P) \hat{H}(1-P),
$$

where the projection operator $P_{\mu}=|\mu\rangle\langle\mu|$ and $P=\sum_{\mu} P_{\mu}$.

In Eq. (2.12) [as well as (2.9)] the Hamiltonian is divided into a bound-state part (first term) and a scattering part (remaining terms) with the understanding that in considering $(1-P) \hat{H}(1-P)$ one ignores the states $|\mu\rangle$ which formally are its eigenfunctions of zero energy. The subsidiary condition (2.11) appears because the Fock space $\mathscr{F}$ was initially expanded to accommodate ideal-state operators $\hat{b}_{\mu}, \hat{b}_{\mu}^{\dagger}$, etc., and merely serves to restrict us to the appropriate subspace of the enlarged space $\mathscr{H}$. There is no analogous condition when considering (2.12).

In the case of realistic scattering or reaction problems where the bound states are composites (atoms, molecules, etc.), the one-particle Fock-Tani representation outlined above can be straightforwardly generalized ${ }^{11}$ and leads naturally to a separation of the Hamiltonian into portions representing physically distinct processes (elastic scattering, inelastic scattering, various rearrangement processes). Such a second-quantization representation also has other advantages over separation based on projection operators, in that powerful field-theoretic techniques previously found useful in electron scattering from atoms ${ }^{12}$ now become applicable to reactive scattering. A review of the field-theoretic formulation of three-particle reactive scattering in terms of the Fock-Tani representation has been given recently by Ficocelli Varracchio. ${ }^{13}$

Normally, imposition of the subsidiary condition (2.11) would make the solution of any practical problem difficuIt and we would have merely traded the difficulty inherent in the noncanonical commutation relations of the realstate operators $\hat{\psi}_{\mu}, \hat{\psi}_{\mu}^{\dagger}$, etc. [see Eq. (2.4)], for something equally difficult. However, an enormous simplification which makes the rigmarole of the Fock-Tani transformation worthwhile occurs if we consider scattering from a time-dependent point of view. At $t \rightarrow-\infty$ we construct a wave packet infinitely far away from the scattering center. It is orthogonal to all bound states by virtue of their finite spatial range, and the subsidiary condition (2.11) is satisfied in the limit $t \rightarrow-\infty$. Now, since $\Sigma_{\mu} \hat{\psi}_{\mu}^{\dagger} \hat{\psi}_{\mu}$ commutes with the Hamiltonian $\mathscr{H}_{\text {FT }}$ (this is easily seen for the corresponding operators $\sum_{\mu} \hat{b}_{\mu}^{+} \hat{b}_{\mu}$ and $\hat{H}_{F}$ before the transformation), Eq. (2.11) is satisfied at all times and may be ignored altogether. This essential simplification is then justified for the time-independent view of scattering in the usual manner.

The Hamiltonian in (2.9) can be divided as usual into an unperturbed part $\hat{H}_{0}$ [one possibility is $\left.\hat{H}_{0}=\Sigma_{\mu} \epsilon_{\mu} \hat{b}_{\mu}^{\dagger} \hat{b}_{\mu}+\int d \mathbf{r} \hat{a}^{\dagger}(\mathbf{r}) T(\mathbf{r}) \hat{a}(\mathbf{r})\right]$ and a perturba- tion $\hat{V}$ and the full scattering function $\left|\chi^{( \pm)}\right\rangle$may be expanded in powers of $\hat{V}$. Since a truncated expansion $\left|\chi_{\mathrm{app}}^{( \pm)}\right\rangle$is defined in terms of the creation operator $\hat{a}^{\dagger}(\mathbf{r})$ only, it is orthogonal to all bound states $\left\{\hat{b}_{\mu}^{+}|0\rangle\right.$, $\mu=1,2, \ldots\}$. This is quite unlike the perturbation expansion in the Fock space where an approximate scattering function is not orthogonal to the bound states and this lack of orthogonality introduces an error in the scattering amplitude which is tolerably small at sufficiently high energies only. Forcing appropriate orthogonality on the approximate scattering states $\left|\chi_{\text {app }}^{( \pm)}\right\rangle$leads to orthogonality corrections to the Fock-space scattering amplitudemathematically these arise from the last term in (2.9) which serve to extend the range of validity of the perturbation approximation to lower energies. In fact, our approach can be regarded as a generalization of Weinberg's "quasiparticle method"14 to which it is closely related both in concept and in the form of orthogonalization corrections. Weinberg showed, within the context of oneparticle scattering from a potential supporting bound states, that such orthogonalization greatly improves the convergence of the Born expansion. We expect a similar benefit in the case of the composite-particle generalization employed here.

An important proviso must be added to the remarks of previous paragraphs. We are justified in ignoring the subsidiary condition (2.11) only as long as we calculate the exact scattering state $\left|\chi^{( \pm)}\right\rangle$. The approximate state $\left|\chi_{\text {app }}^{( \pm)}\right\rangle$does not lie entirely in the Fock-Tani subspace $\mathscr{H}_{\text {FT }}$ but contains an admixture of states outside $\mathscr{H}_{\text {FT }}$. Thus, truncation of the perturbation expansion for $\left|\chi^{( \pm)}\right\rangle$ in the Fock-Tani space introduces an error in the scattering amplitude analogous to the error in the truncated Fock-space amplitude. It is not possible to make any definite general statement about the relative magnitudes of these errors. However, such numerical evidence as is already available ${ }^{2}$ indicates that this representation can yield rather accurate results for rearrangement processes even in first order. We take this as adequate justification for use of this approach herein. Additional a posteriori evidence is supplied by the accuracy of our calculated cross sections.

\section{FOCK-TANI TRANSFORMATION FOR REARRANGEMENT COLLISIONS:

$$
1+(23) \rightarrow(12)+3
$$

A similar transformation was previously considered by Girardeau $^{15}$ for scattering of positrons from hydrogen atoms. In that case the transformation was somewhat simplified because the nuclear coordinate was frozen by virtue of the (supposedly) infinite mass of the nucleus. In the more general case of charge transfer, one may still eliminate a coordinate from consideration; the center-ofmass coordinate is the most convenient choice. We therefore introduce elementary annihilation and creation operators $\left\{\hat{a}_{i}\left(\mathbf{r}_{i}\right), \hat{a}_{i}^{\dagger}\left(\mathbf{r}_{i}\right), i=1,2,3\right\}$ for the particles 1,2 , and 3 . These are assumed to be distinguishable so that we may require $\hat{a}_{i}\left(\mathbf{r}_{i}\right), \hat{a}_{j}^{\dagger}\left(\mathbf{r}_{j}\right)$, etc. to satisfy boson commutation relations.

Assuming pairwise interaction, the Fock Hamiltonian is 


$$
\begin{aligned}
\hat{H}_{F}=\sum_{i=1}^{3} \int d \mathbf{r}_{i} \hat{a}_{i}^{\dagger}\left(\mathbf{r}_{i}\right) T_{i}\left(\mathbf{r}_{i}\right) \widehat{a}_{i}\left(\mathbf{r}_{i}\right) \\
+\sum_{i=1}^{3} \int d \mathbf{r}_{i} d \mathbf{r}_{i+1} \hat{a}_{i}^{\dagger}\left(\mathbf{r}_{i}\right) \hat{a}_{i+1}^{\dagger}\left(\mathbf{r}_{i+1}\right) \\
\quad \times V_{i, i+1}\left(\mathbf{r}_{i}, \mathbf{r}_{i+1}\right) \hat{a}_{i+1}\left(\mathbf{r}_{i+1}\right) \hat{a}_{i}\left(\mathbf{r}_{i}\right),
\end{aligned}
$$

where the subscript 4 is to be regarded as 1 .

The interactions $V_{12}$ and $V_{23}$ are assumed sufficiently strong to support a number of bound states. In analogy with Eq. (2.3) we introduce the following bound-state creation operators:

$$
\begin{aligned}
&\left(\hat{\psi}_{\mu_{\alpha}}^{\alpha}\right)^{\dagger}=(2 \pi)^{-3 / 2} \int d \mathbf{r}_{i} d \mathbf{r}_{j} \psi_{\bar{\mu}_{\alpha}}^{\alpha}\left(\mathbf{r}_{\alpha}\right) \\
& \times \exp \left(i \mathbf{k}_{\alpha} \cdot \mathbf{R}_{\alpha}\right) \hat{a}_{i}^{\dagger}\left(\mathbf{r}_{i}\right) \hat{a}_{j}^{\dagger}\left(\mathbf{r}_{j}\right), \\
& \alpha \equiv(i j)=(12) \text { or }(23) .
\end{aligned}
$$

In our notation, the superscript $\alpha=(i j)$ labels the pair of particles, $\mathbf{r}_{\alpha}$ and $\mathbf{R}_{\alpha}$ are the relative and center-of-mass coordinates of the pair, and $\mu_{\alpha}$ is a collective label for the internal state $\bar{\mu}_{\alpha}$ and the center-of-mass momentum $\mathbf{k}_{\alpha}$. Annihilation operators are defined by Hermitian conjugation of (3.2). The commutation relations of these operators are obtained from the commutation relations for the elementary operators $\hat{a}_{i}^{\dagger}\left(\mathbf{r}_{i}\right), \hat{a}_{j}\left(\mathbf{r}_{j}\right)$, etc.

We next introduce "ideal-state" operators $\hat{b}_{\mu}^{\alpha}$ and $\left(\hat{b}_{\mu}^{\alpha}\right)^{\dagger}$ for $\alpha=(12)$ and (23) and define the following unitary operators in analogy with Eq. (2.8):

$$
\hat{U}^{\alpha}=\exp \left(\frac{\pi}{2} \sum_{\mu_{\alpha}}\left[\left(\hat{\psi}_{\mu_{\alpha}}^{\alpha}\right)^{\dagger} \hat{b}_{\mu_{\alpha}}^{\alpha}-\left(\hat{b}_{\mu_{\alpha}}^{\alpha}\right)^{\dagger} \hat{\psi}_{\mu_{\alpha}}^{\alpha}\right]\right) .
$$

The Fock-Tani transformation is now made by successive application of the transformations generated by $\hat{U}^{(12)}$ and $\hat{U}^{(23)}$, but unlike (2.9) there are now two possibilities. Alternative transformations,

$$
\hat{H}_{\mathrm{FT} 1}=\left(\hat{U}^{(23)}\right)^{-1}\left(\hat{U}^{(12)}\right)^{-1} \hat{H}_{F} \hat{U}^{(12)} \hat{U}^{(23)}
$$

and

$$
\hat{H}_{\mathrm{FT} 2}=\left(\hat{U}^{(12)}\right)^{-1}\left(\hat{U}^{(23)}\right)^{-1} \hat{H}_{F} \hat{U}^{(23)} \hat{U}^{(12)},
$$

are not identical because $\left[\hat{U}^{(12)}, \hat{U}^{(23)}\right] \neq 0$. In this paper we work with (3.4a) because for the reaction under consideration, $1+(23) \rightarrow(12)+3$, the corresponding firstorder amplitude is easily interpreted; it is a sum of the first-order Fock amplitude and an orthogonality correction, much as in the case of potential scattering. The zeroth-order wave functions for all possible initial and final states are now orthogonal, thus satisfying the same orthogonality relation as the exact scattering states. The first- and higher-order wave functions are, however, not exactly orthogonal due to the possibility of rearrangement, but exact orthogonality is restored at infinite order. We leave the investigation of the implication of this for future work when we shall extend our analysis to higher perturbative corrections. We note here only that the orthogonality obtaining in first order in our approach is a distinct improvement over approaches in which such orthogonali- ty is violated.

The transformed Hamiltonian is rather lengthy to write and we have relegated the complete expression to Appendix A. Parts of the Hamiltonian relevant to this paper are the zeroth-order Hamiltonian

$\hat{H}_{0}=\sum_{\alpha, \mu_{\alpha}} \epsilon_{\mu_{\alpha}}^{\alpha}\left(\hat{b}_{\mu_{\alpha}}^{\alpha}\right)^{\dagger} \hat{b}_{\mu_{\alpha}}^{\alpha}+\sum_{i=1}^{3} \int d \mathbf{r}_{i} \hat{a}_{i}^{\dagger}\left(\mathbf{r}_{i}\right) T_{i}\left(\mathbf{r}_{i}\right) \hat{a}_{i}\left(\mathbf{r}_{i}\right)$

and the rearrangement interaction

$$
\begin{aligned}
V_{\text {rearr }}=\sum_{\mu_{\alpha_{1}}, \mu_{\alpha_{2}}} \int & d \mathbf{r}_{3} d \mathbf{r}_{1}\left(\hat{b}_{\mu_{12}}^{(12)}\right)^{\dagger} \hat{a}_{3}^{\dagger}\left(\mathbf{r}_{3}\right)\left\langle\mu_{12} \mathbf{r}_{3}|V| \mu_{23} \mathbf{r}_{1}\right\rangle \\
& \times \widehat{a}_{1}\left(\mathbf{r}_{1}\right) \hat{b}_{\mu_{12}}^{(12)}+\text { H.c. }
\end{aligned}
$$

where the interaction $\left\langle\mu_{12} \mathbf{r}_{3}|V| \mu_{23} \mathbf{r}_{1}\right\rangle$ is given in (A5) and H.c. denotes Hermitian conjugation. The first-order approximation to the $T$ matrix is then given by the matrix element of $V_{\text {rearr }}$ between appropriate eigenstates of $\hat{H}_{0}$.

\section{FIRST-ORDER T-MATRIX ELEMENT FOR REARRANGEMENT}

We will use three alternative sets of coordinates, shown in Fig. 2: (i) $\mathbf{r}_{1}, \mathbf{r}_{2}$, and $\mathbf{r}_{3}$, which are coordinates of the particles 1, 2, and 3, respectively, in a fixed coordinate system; (ii) $\mathbf{R}$ (center-of-mass coordinate), $\mathbf{r}_{3 \alpha_{1}}$ [vector from particle 3 to the center of mass of $(1,2)$ ], and $\mathbf{r}_{12}$ [relative coordinate of $(1,2)$ ]; and (iii) $\mathbf{R}, \mathbf{r}_{\alpha_{2} 1}$ [vector from the center of mass of $(2,3)$ to particle 1] and $r_{23}$ [relative coordinate of $(2,3)]$.

The initial and final-state wave functions

$$
\begin{aligned}
\left|\psi_{i}\right\rangle=(2 \pi)^{-3 / 2} \int & d \mathbf{r}_{1}^{\prime} \exp \left(i \mathbf{k}_{1} \cdot \mathbf{r}_{1}^{\prime}\right) \\
& \times \hat{a}_{1}^{\dagger}\left(\mathbf{r}_{1}^{\prime}\right)\left(\hat{b}_{\mu_{23}}^{(23)}\right)^{\dagger}|0\rangle
\end{aligned}
$$

and

$$
\begin{aligned}
\left|\psi_{f}\right\rangle=(2 \pi)^{-3 / 2} \int & d \mathbf{r}_{3}^{\prime} \exp \left(i \mathbf{k}_{3} \cdot \mathbf{r}_{3}^{\prime}\right) \\
& \times \widehat{a}_{3}^{\dagger}\left(\mathbf{r}_{3}^{\prime}\right)\left(\hat{b}_{v_{12}}^{(12)}\right)^{\dagger}|0\rangle
\end{aligned}
$$

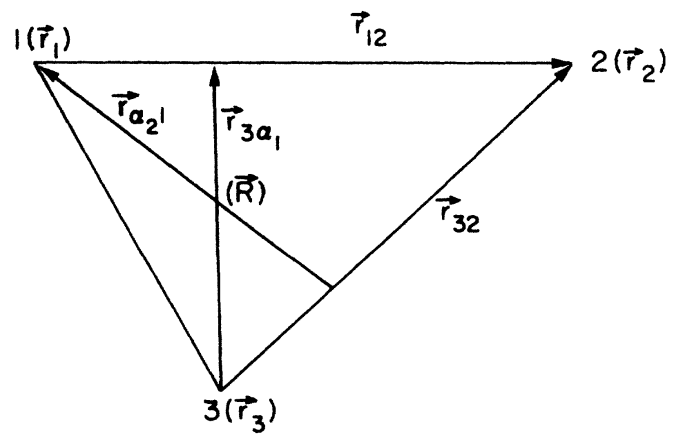

FIG. 2. Alternative coordinates for the three-particle system. The vector from the origin (not shown in the figure) to a specified point is indicated in parentheses beside the point in question. Thus, $r_{1}$ is the vector from the origin to particle 1 , etc. 
are eigenfunctions of $\hat{H}_{0}$ and represent plane waves of $\bar{\alpha}$ impinging on bound states of $\alpha$. We recall that the label $\mu_{\alpha_{1}}$ includes the internal state $\bar{\mu}$ of the pair $\alpha_{1}$ and its center-of-mass momentum $\mathbf{k}_{\mu}$. by

The first-order $T$-matrix element is given quite simply

$$
\begin{aligned}
T_{v_{12} \leftarrow \mu_{23}}^{(1)}=\left\langle\psi_{f}\left|V_{\text {rearr }}\right| \psi_{i}\right\rangle & \\
= & =(2 \pi)^{-3} \int d \mathbf{r}_{3} d \mathbf{r}_{1} e^{-i \mathbf{k}_{3} \cdot \mathbf{r}_{3}} \\
& \times\left\langle v_{12} \mathbf{r}_{3}|V| \mu_{23} \mathbf{r}_{1}\right\rangle e^{i \mathbf{k}_{1} \cdot \mathbf{r}_{1}}
\end{aligned}
$$

and splits into a sum of two terms arising from the $\left[\delta\left(\mathbf{r}_{1}-\mathbf{r}_{1}^{\prime}\right) \delta\left(\mathbf{r}_{2}-\mathbf{r}_{2}^{\prime}\right)-\Delta^{12}\left(\mathbf{r}_{1}^{\prime}, \mathbf{r}_{2}^{\prime} ; \mathbf{r}_{1}, \mathbf{r}_{2}\right)\right]$ factor in the definition of $\left\langle\mu_{12} \mathbf{r}_{3}|V| \mu_{23} \mathbf{r}_{1}\right\rangle$ [see Eq. (A5)]. It will emerge later that the first term gives the Jackson-Schiff-Bates Dalgarno (JSBD) approximation to the $T$ matrix and the second term represents orthogonality corrections characteristic of the present formalism. Thus

$$
T_{v_{12} \leftarrow \mu_{23}}^{(1)}=T_{v_{12} \leftarrow \mu_{23}}^{\mathrm{JSBD}}+T_{v_{12} \leftarrow \mu_{23}}^{\text {ortho }} .
$$

In substituting (A5) into (4.2) we note that

$$
\psi_{v_{12}}^{(12)}\left(\mathbf{r}_{1}, \mathbf{r}_{2}\right)=(2 \pi)^{-3 / 2} \exp \left(i \mathbf{k}_{v} \cdot \mathbf{R}_{12}\right) \psi_{\bar{v}}^{(12)}\left(\mathbf{r}_{12}\right)
$$

and

$$
\psi_{\mu_{23}}^{(23)}\left(\mathbf{r}_{2}, \mathbf{r}_{3}\right)=(2 \pi)^{-3 / 2} \exp \left(i \mathbf{k}_{\mu} \cdot \mathbf{R}_{23}\right) \psi_{\bar{\mu}}^{(23)}\left(\mathbf{r}_{23}\right) .
$$

In this equation $\mathbf{k}_{\mu}$ and $\mathbf{k}_{v}$ are the center-of-mass momenta of the pairs $\alpha_{2}=(23)$ and $\alpha_{1}=(12)$, respectively. As a first step in factoring the overall center-of-mass momentum, we replace the momenta $\mathbf{k}_{1}$ and $\mathbf{k}_{\mu}$ by $\mathbf{k}_{i}$ [relative momentum of particle 1 and pair (23)] and the overall center-of-mass momentum $\mathbf{K}_{i}$,

$$
\mathbf{K}_{i}=\mathbf{k}_{1}+\mathbf{k}_{\mu}
$$

and

$$
\mathbf{k}_{i}=\left[\left(m_{2}+m_{3}\right) \mathbf{k}_{1}-m_{1} \mathbf{k}_{\mu}\right] /\left(m_{1}+m_{2}+m_{3}\right),
$$

where $m_{i}$ denotes the mass of the $i$ th particle. Similarly, the pair $\left(\mathbf{k}_{3}, \mathbf{k}_{\boldsymbol{v}}\right)$ is replaced by $\left(\mathbf{k}_{f}, \mathbf{K}_{f}\right)$ with obvious physical significance.

The first term $T_{v_{12} \leftarrow \mu_{23}}^{\mathrm{JSBD}}$, which is written as an integral over $\mathbf{r}_{1}, \mathbf{r}_{2}$, and $\mathbf{r}_{3}$, can be simplified by switching to $\left(\mathbf{R}, \mathbf{r}_{3 \alpha_{1}}, \mathbf{r}_{12}\right)$ coordinates. Integration over the center-ofmass coordinate $\mathbf{R}$ gives a factor $\delta\left(\mathbf{K}_{i}-\mathbf{K}_{f}\right)$ which expresses conservation of center-of-mass momentum.

The final result

$$
\begin{aligned}
T_{v_{12} \leftarrow \mu_{23}}^{\mathrm{JSBD}}=(2 \pi)^{-3} \delta\left(\mathbf{K}_{i}-\mathbf{K}_{f}\right) \int & d \mathbf{r}_{3 \alpha_{1}} d \mathbf{r}_{12} \exp \left(-i \mathbf{k}_{f} \cdot \mathbf{r}_{3 \alpha_{1}}\right) \\
& \times\left[\psi_{\bar{v}}^{(12)}\left(\mathbf{r}_{12}\right)\right]^{*}\left[V_{13}\left[\mathbf{r}_{3 \alpha_{1}}-\frac{m_{2}}{m_{1}+m_{2}} \mathbf{r}_{12}\right)+V_{23}\left[\mathbf{r}_{3 \alpha_{1}}+\frac{m_{1}}{m_{1}+m_{2}} \mathbf{r}_{12}\right)\right] \\
& \times \psi_{\bar{\mu}}^{(23)}\left[\mathbf{r}_{3 \alpha_{1}}+\frac{m_{1}}{m_{1}+m_{2}} \mathbf{r}_{12}\right] \exp \left[i \mathbf{k}_{i} \cdot\left(\frac{m_{3}}{m_{2}+m_{3}} \mathbf{r}_{3 \alpha_{1}}-\frac{m_{2}\left(m_{1}+m_{2}+m_{3}\right)}{\left(m_{1}+m_{2}\right)\left(m_{2}+m_{3}\right)} \mathbf{r}_{12}\right)\right]
\end{aligned}
$$

is just the matrix element considered by Jackson and Schiff ${ }^{6}$ and Bates and Dalgarno. ${ }^{7}$ In Eq. (4.6) we have written coordinates $\mathbf{r}_{13}, \mathbf{r}_{23}$, and $\mathbf{r}_{\alpha_{2} 1}$ explicitly in terms of integration variables $\mathbf{r}_{3 \alpha_{1}}$ and $\mathbf{r}_{12}$ by referring to Fig. 2 .

From Eq. (4.2), the second term $T_{v_{12} \leftarrow \mu_{23}}^{\text {ortho }}$ is given as an integral over the coordinates $\mathbf{r}_{1}, \mathbf{r}_{2}, \mathbf{r}_{3}, \mathbf{r}_{1}^{\prime}$, and $\mathbf{r}_{2}^{\prime}$. The first step in its simplification is to replace $\left(\mathbf{r}_{1}, \mathbf{r}_{2}\right)$ by the relative and center-of-mass coordinates $\left(\mathbf{r}_{12}, \mathbf{R}_{12}\right)$ for the pair (12) and $\left(\mathbf{r}_{1}^{\prime}, \mathbf{r}_{2}^{\prime}\right)$ by $\left(\mathbf{r}_{12}^{\prime}, \mathbf{R}_{12}^{\prime}\right)$. The bound-state kernel,

$$
\Delta^{(12)}\left(\mathbf{r}_{1}^{\prime}, \mathbf{r}_{2}^{\prime} ; \mathbf{r}_{1}, \mathbf{r}_{2}\right)=\delta\left(\mathbf{R}_{12}^{\prime}-\mathbf{R}_{12}\right) \sum_{\lambda} \psi_{\lambda}^{(12)}\left(\mathbf{r}_{12}^{\prime}\right)\left[\psi_{\lambda}^{(12)}\left(\mathbf{r}_{12}\right)\right]^{*}
$$

is then seen to contain a $\delta$ function and integration over $\mathbf{R}_{12}$ is immediate. We then revert to $\left(\mathbf{r}_{1}^{\prime}, \mathbf{r}_{2}^{\prime}\right)$ before introducing new coordinates $\left(\mathbf{R}^{\prime}, \mathbf{r}_{3 \alpha_{1}}^{\prime}, \mathbf{r}_{12}^{\prime}\right)$ instead of $\left(\mathbf{r}_{1}^{\prime}, \mathbf{r}_{2}^{\prime}, \mathbf{r}_{3}\right)$ in analogy with $\left(\mathbf{R}, \mathbf{r}_{3 \alpha_{1}}, \mathbf{r}_{12}\right)$ in Fig. 2. Integration over $\mathbf{R}^{\prime}$ then yields a $\delta$ function in initial and final center-of-mass momenta $\mathbf{K}_{i}$ and $\mathbf{K}_{f}$. The final form of the orthogonality correction is

$$
\begin{aligned}
T_{v_{12} \leftarrow \mu_{23}}^{\text {ortho }}=-(2 \pi)^{-3} \delta\left(\mathbf{K}_{i}-\mathbf{K}_{f}\right) \sum_{\lambda} \int & d \mathbf{r}_{12} d \mathbf{r}_{3 \alpha_{1}}^{\prime} d \mathbf{r}_{12}^{\prime} \exp \left(-i \mathbf{q}_{1} \cdot \mathbf{r}_{3 \alpha_{1}}^{\prime}\right)\left[\psi_{\bar{v}}^{(12)}\left(\mathbf{r}_{12}^{\prime}\right)\right]^{*} \\
& \times\left[V_{13}\left[\mathbf{r}_{3 \alpha_{1}}^{\prime}-\frac{m_{2}}{m_{1}+m_{2}} \mathbf{r}_{12}^{\prime}\right]+V_{23}\left[\mathbf{r}_{3 \alpha_{1}}^{\prime}+\frac{m_{1}}{m_{1}+m_{2}} \mathbf{r}_{12}^{\prime}\right)\right] \\
& \times \psi_{\lambda}^{(12)}\left(\mathbf{r}_{12}^{\prime}\right)\left[\psi_{\lambda}^{(12)}\left(\mathbf{r}_{12}\right)\right]^{*} \exp \left(-i \mathrm{~m} \mathbf{k}_{i} \cdot \mathbf{r}_{12}\right) \psi_{\bar{\mu}}^{(23)}\left[\mathbf{r}_{3 \alpha_{1}}^{\prime}+\frac{m_{1}}{m_{1}+m_{2}} \mathbf{r}_{12}\right)
\end{aligned}
$$


with

$$
\mathbf{q}_{1}=\mathbf{k}_{f}-\frac{m_{3}}{m_{2}+m_{3}} \mathbf{k}_{i}
$$

and

$$
\mathbf{m}=\frac{m_{2}\left(m_{1}+m_{2}+m_{3}\right)}{\left(m_{1}+m_{2}\right)\left(m_{2}+m_{3}\right)} .
$$

In this expression $\lambda$ ranges over all bound states of the pair (12) and all coordinates have been written in terms of integration variables with reference to Fig. 2.

The orthogonality correction can be interpreted in two alternative ways. It arises from a potential, say $V_{\text {nonre }}$, which must be subtracted from the overall potential to give the rearrangement part of the interaction, $V_{\text {rearr }}$. $V_{\text {nonre }}$ consists of a sum of terms, each associated with a bound state of (12). The $\lambda=\bar{v}$ term, i.e., when the intermediate state is the same as the final bound state of the composite (12), is just the static interaction in the final channel. Remaining terms, arising from other intermediate states, give nonlocal contributions to $V_{\text {nonre }}$. Alternatively, $T_{v_{12} \leftarrow \mu_{23}}^{\text {ortho }}$ can be regarded as correcting for the lack of orthogonality between the initial state and all possible final states, the $\lambda=\bar{v}$ term corresponding to the particular final state in question.

In the special case of charge transfer, particles 1 and 3 are nuclei and particle 2 is an electron. Then $m_{2} \ll m_{1}$, $m_{3}$ and $m_{1} \sim m_{3}$. We note that it is a good approximation to neglect all terms of order $m_{e} / m_{p}$ unless they are multiplied by a nuclear momentum $k$ which may be large.

$T_{v_{12} \leftarrow \mu_{23}}^{\mathrm{JSBD}}$ also consists of two terms. The second term

$$
V_{23}\left[\mathbf{r}_{3 \alpha_{1}}+\frac{m_{1}}{m_{1}+m_{2}} \mathbf{r}_{12}\right]\left[=V_{23}\left(\mathbf{r}_{23}\right)\right]
$$

alone constitutes the Oppenheimer-Brinkman-Kramers (OBK) approximation. ${ }^{3,4}$ The first term

$$
V_{13}\left[\mathbf{r}_{3 \alpha_{1}}-\frac{m_{2}}{m_{1}+m_{2}} \mathbf{r}_{12}\right]=V_{13}\left(\mathbf{r}_{13}\right)
$$

$$
\begin{aligned}
T_{v_{12} \leftarrow \mu_{23}}^{\mathrm{OBK}}=(2 \pi)^{-3} \int & d \mathbf{r}_{3 \alpha_{1}} d \mathbf{r}_{12} \exp \left(-i \mathbf{q}_{1} \cdot \mathbf{r}_{3 \alpha_{1}}\right)\left[\psi_{\bar{v}}^{12}\left(\mathbf{r}_{12}\right)\right]^{*} \\
& \times V_{23}\left(\mathbf{r}_{3 \alpha_{1}}+\frac{m_{1}}{m_{1}+m_{2}} \mathbf{r}_{12}\right) \psi_{\bar{\mu}}^{23}\left[\mathbf{r}_{3 \alpha_{1}}+\frac{m_{1}}{m_{1}+m_{2}} \mathbf{r}_{12}\right) \exp \left(-i \mathbf{m} \mathbf{k}_{i} \cdot \mathbf{r}_{12}\right)
\end{aligned}
$$

and

$$
\begin{array}{r}
T_{v_{12} \leftarrow \mu_{23}}^{\mathrm{ortho}}=-(2 \pi)^{-3} \sum_{\lambda} \int d \mathbf{r}_{12} d \mathbf{r}_{3 \alpha_{1}}^{\prime} d \mathbf{r}_{12}^{\prime} \exp \left(-i \mathbf{q}_{1} \cdot \mathbf{r}_{3 \alpha_{1}}\right)\left[\psi_{\bar{v}}^{(12)}\left(\mathbf{r}_{12}^{\prime}\right)\right]^{*} V_{23}\left(\mathbf{r}_{3 \alpha_{1}}^{\prime}+\frac{m_{1}}{m_{1}+m_{2}} \mathbf{r}_{12}^{\prime}\right) \\
\quad \times \psi_{\lambda}^{(12)}\left(\mathbf{r}_{12}^{\prime}\right)\left[\psi_{\lambda}^{(12)}\left(\mathbf{r}_{12}\right)\right]^{*} \exp \left(-i \mathbf{m} \mathbf{k}_{i} \cdot \mathbf{r}_{12}\right) \psi_{\bar{\mu}}^{(23)}\left(\mathbf{r}_{3 \alpha_{1}}^{\prime}+\frac{m_{1}}{m_{1}+m_{2}} \mathbf{r}_{12}\right) .
\end{array}
$$

Equations (5.2a) and (5.2b) for $T_{v_{12} \leftarrow \mu_{23}}^{\mathrm{OBK}}$ and $T_{v_{12} \leftarrow \mu_{23}}^{\text {ortho }}$ are obtained from Eqs. (4.6) and (4.7) for $T_{v_{12} \leftarrow \mu_{23}}^{\mathrm{JSBD}}$ and $T_{v_{12} \leftarrow \mu_{23}}^{\text {ortho }}$, respectively, by dropping the internuclear potential. We have also suppressed the $\delta$ function denoting conservation of overall center-of-mass momentum. represents internuclear interaction and it is very nearly canceled by the first term in (4.7). To see this, note that to a very good approximation we can replace

$$
V_{13}\left[\mathbf{r}_{3 \alpha_{1}}^{\prime}-\frac{m_{2}}{m_{1}+m_{2}} \mathbf{r}_{12}^{\prime}\right) \rightarrow V_{13}\left(\mathbf{r}_{3 \alpha_{1}}^{\prime}\right)
$$

in Eq. (4.7) and also make a corresponding approximation . Integration over $\mathbf{r}_{12}^{\prime}$ in the first term of (4.7) then bution in the sum over $\lambda$. Substituting (4.8a) and (4.8b) for $\mathbf{q}_{1}$ and $\mathbf{m}$, respectively, in the resulting expression, a qearrangement of terms and comparison with the the approximation (4.9), we incur a small error of order $m_{2} /\left(m_{1}+m_{2}\right) \sim m_{e} / m_{p}$ so that the cancellation is not exbut nearly so. In light of the observation made by potential makes a negligible contribution of order $m_{e} / m_{p}$ to the exact reaction amplitude, our result for the corresponding first-order amplitude is very satisfying. In subclear repulsion.

The role of internuclear potential in charge transfer has received much attention and it has been long known that one must correct for the lack of orthogonality between the Bassel and Gerguoy ${ }^{16}$ achieved this limited objective by subtracting the static interaction in the final channel from total interaction in the post form of the transition ma(4.8), this is a special case of our result which we have obtained starting from a much more general objective of considering scattering in the presence of bound states.

\section{EVALUATION OF THE $T$ MATRIX}

It follows from the discussion of Sec. IV that to a very good approximation the $T$-matrix element is given by

$$
T_{v_{12} \leftarrow \mu_{23}}=T_{v_{12} \leftarrow \mu_{23}}^{\mathrm{OBK}}+T_{v_{12} \leftarrow \mu_{23}}^{\text {ortho }},
$$

where 
$T_{v_{12} \leftarrow \mu_{23}}^{\mathrm{OBK}}$ is easily evaluated by reverting to independent variables $r_{12}$ and $r_{32}$. We quote the final result from Appendix I of Jackson and Schiff ${ }^{6}$ after transcribing it into our notation. It is first necessary to define another momentum-transfer vector

$$
\mathbf{q}_{2}=\mathbf{k}_{i}-\frac{m_{1}}{m_{1}+m_{2}} \mathbf{k}_{f}
$$

analogous to $q_{1}$ defined by Eq. (4.8a). Then

$$
T_{v_{12} \leftarrow \mu_{23}}^{\mathrm{OBK}}=-\left(\frac{m_{1}+m_{2}}{2 m_{1} m_{2}} q_{2}^{2}+\epsilon_{\bar{v}}\right) \Phi_{\bar{\mu}}\left(-\mathbf{q}_{1}\right) \Phi_{\bar{v}}^{*}\left(\mathbf{q}_{2}\right),
$$

where $\epsilon_{\bar{v}}$ is the binding energy of the pair (12) in the internal state $\bar{v}$ and $\Phi_{\bar{\mu}}\left(-q_{1}\right)$ and $\Phi_{\bar{v}}\left(q_{2}\right)$ are momentum-space wave functions.

In order to evaluate the orthogonality correction, we first rewrite the coordinate-space wave functions $\left[\psi_{\lambda}^{\alpha_{1}}\left(\mathbf{r}_{12}\right)\right]^{*}$ and $\psi_{\mu}^{\alpha_{2}}\left(\mathbf{r}_{3 \alpha_{1}}^{\prime}+\left(m_{1} / m_{1}+m_{2}\right) \mathbf{r}_{12}\right)$ as Fourier

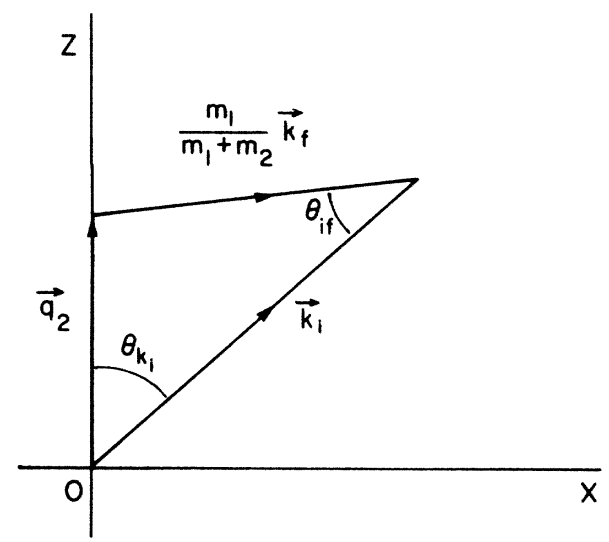

FIG. 3. Spatial arrangement of vectors $\mathbf{k}_{i}, \mathbf{k}_{f}$, and $\mathbf{q}_{2}$ for the evaluation of the integral in Eq. (5.6).

transforms of corresponding momentum-space wave functions $\Phi_{\lambda}^{*}(\mathbf{p})$ and $\Phi_{\bar{\mu}}\left(\mathbf{p}_{1}\right)$ and also rewrite the Coulomb interaction as a Fourier transform,

$$
\left.\left|\mathbf{r}_{3 \alpha_{1}}^{\prime}+\frac{m_{1}}{m_{1}+m_{2}} \mathbf{r}_{12}\right|^{-1}=\frac{1}{2 \pi^{2}} \int d \mathbf{p}_{2} \frac{1}{p_{2}^{2}} \exp \left[-i \mathbf{p}_{2} \cdot \mid \mathbf{r}_{3 \alpha_{1}}^{\prime}+\frac{m_{1}}{m_{1}+m_{2}} \mathbf{r}_{12}\right]\right] \text {. }
$$

This introduces three new integration variables $\mathbf{p}, \mathbf{p}_{1}$, and $\mathbf{p}_{2}$, but integration over $\mathbf{r}_{3 \alpha_{1}}$ and $\mathbf{r}_{12}$ immediately gives a delta function each, $\delta\left(\mathbf{q}_{1}+\mathbf{p}_{2}-\mathbf{p}_{1}\right)$ and $\delta\left(\mathbf{p}-\left(m_{1} / m_{1}+m_{2}\right) \mathbf{p}_{1}+\mathbf{m} \mathbf{k}_{i}\right)$. This allows us to integrate over $\mathbf{p}_{1}$ and $\mathbf{p}_{2}$. After some simple algebraic rearrangement to show that $\mathbf{m} \mathbf{k}_{i}-\left(m_{1} / m_{1}+m_{2}\right) \mathbf{q}_{1}=\mathbf{q}_{2}$, the orthogonality correction is rewritten as

$$
\begin{aligned}
T_{v_{12} \leftarrow \mu_{23}}^{\text {ortho }}=-\frac{Z_{2} Z_{3}}{2 \pi^{2}} \frac{m_{1}+m_{2}}{m_{1}} \sum_{\lambda} \int & d \mathbf{p} d \mathbf{r}_{12}^{\prime} \frac{1}{\left|\mathbf{p}+\mathbf{q}_{2}\right|^{2}} \exp \left[-i\left(\mathbf{p}+\mathbf{q}_{2}\right) \cdot \mathbf{r}_{12}^{\prime}\right] \\
& \times\left[\psi_{\bar{\nu}}^{(12)}\left(\mathbf{r}_{12}^{\prime}\right)\right]^{*} \psi_{\lambda}^{(12)}\left(\mathbf{r}_{12}^{\prime}\right) \Phi_{\lambda}^{*}(\mathbf{p}) \Phi_{\bar{\mu}}\left(\frac{m_{1}+m_{2}}{m_{1}}\left(\mathbf{p}+\mathbf{m} \mathbf{k}_{i}\right)\right) .
\end{aligned}
$$

In the following discussion we assume without loss of generality that the vector $\mathbf{q}_{2}$ lies along the $z$ axis and $\mathbf{k}_{i}$ and $\mathbf{k}_{f}$ lie in the $x-z$ plane (Fig. 3). For specific final state $\bar{v}$ and intermediate state $\lambda$, the integral

$$
I_{\bar{v} \lambda}\left(\mathbf{p}+\mathbf{q}_{2}\right)=\int d \mathbf{r}_{12}^{\prime} \exp \left[-i\left(\mathbf{p}+\mathbf{q}_{2}\right) \cdot \mathbf{r}_{12}^{\prime}\right]\left[\psi_{\bar{v}}^{\alpha_{1}}\left(\mathbf{r}_{12}^{\prime}\right)\right]^{*} \psi_{\lambda}^{\alpha_{1}}\left(\mathbf{r}_{12}^{\prime}\right)
$$

which appears in (5.6) can be evaluated analytically. (Particular results for the $1 s$ final state and $1 s, 2 s$, and $2 p$ intermediate states are given in Appendix B.) Moreover, for a spherically symmetric final state (i.e., $l_{\bar{v}}=0$ ), it factors into a spherical harmonic and a part depending on $\left|\mathbf{p}+\mathbf{q}_{2}\right|$ only:

$$
I_{\bar{v} \lambda}\left(\mathbf{p}+\mathbf{q}_{2}\right)_{l_{\bar{v}}=0}=I_{\bar{v} \lambda}\left(\left|\mathbf{p}+\mathbf{q}_{2}\right|\right)_{l_{\bar{v}}=0} Y_{l_{\lambda} m_{\lambda}}\left(\Omega_{\mathrm{p}+\mathbf{q}_{2}}\right) \text {. }
$$

We can then sum over $m_{\lambda}$ on the right-hand side of (5.6)-the sum over $\lambda$ is really a triple sum over $n_{\lambda}, l_{\lambda}$, and $m_{\lambda}-$ and use the addition theorem for spherical harmonics to obtain

$$
\sum_{m_{\lambda}} Y_{l_{\lambda} m_{\lambda}}^{*}\left(\Omega_{\mathbf{p}}\right) Y_{l_{\lambda} m_{\lambda}}\left(\Omega_{\mathrm{p}+\mathbf{q}_{2}}\right)=\frac{2 l_{\lambda}+1}{4 \pi} P_{l_{\lambda}}\left(\frac{\mathbf{p} \cdot\left(\mathbf{p}+\mathbf{q}_{2}\right)}{p\left|\mathbf{p}+\mathbf{q}_{2}\right|}\right) \text {. }
$$

Note that with the assumption that $\mathbf{q}_{2}$ lies along the $z$ axis, the argument of the Legendre polynomial depends on $p$ and $\theta_{p}$ but does not depend on the azimuthal angle $\phi_{p}$.

The only $\phi_{p}$ dependence of the integrand in (5.6) comes from the initial-state wave function $\Phi_{\bar{\mu}}\left(\left(m_{1}+m_{2} / m_{1}\right)\right.$ $\left.\times\left(\mathbf{p}+\mathbf{m} \mathbf{k}_{i}\right)\right)$. For a spherically symmetric state $\left(l_{\bar{\mu}}=0\right)$ it is contained in the $\mathbf{p} \cdot \mathbf{k}_{i}$ term in the argument; moreover, for the $1 s$ initial state, integration over $\phi_{p}$ can be done analytically (see Appendix B).

These manipulations reduce the orthogonality correction to its final form which is used for numerical calculation: 

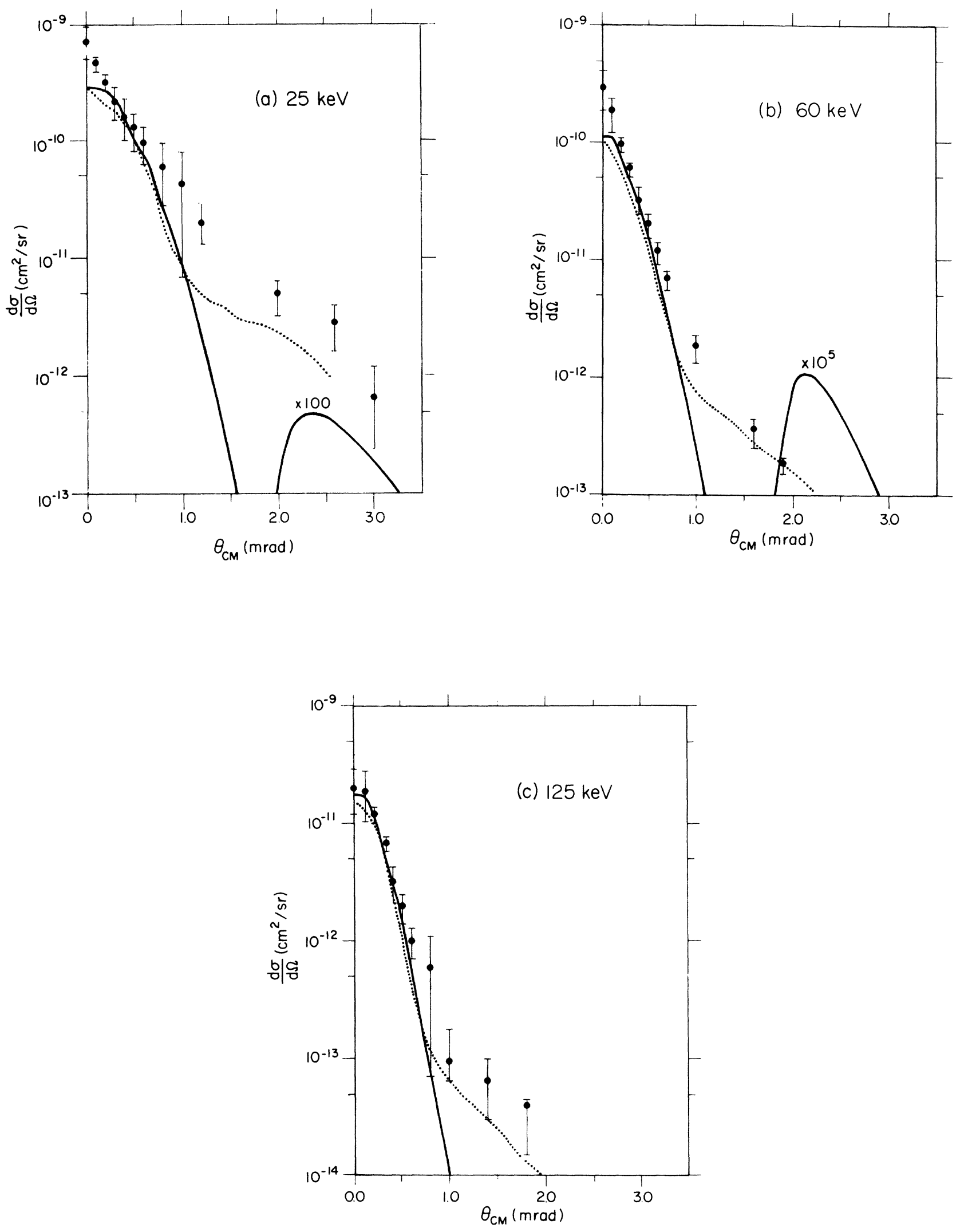

FIG. 4. Center-of-mass differential cross section for resonant charge transfer, $p+\mathbf{H}(1 s) \rightarrow \mathbf{H}(1 s)+p$, at (a) 25 , (b) 60 , and (c) 125 $\mathrm{keV}$. The experimental points are from Martin et al. (Ref. 17), the dotted curve is from the two-state atomic expansion calculation quoted by Martin et al., and the solid curve is the Fock-Tani result with orthogonality corrections due to $1 s, 2 s$, and $2 p$ intermediate states. Note that the experimental cross section is for capture into all bound states and it is expected to be $20 \%$ greater than the cross section for capture into the ground state. The secondary peak in (a) and (b) has been scaled up by the indicated factor. 


$$
\begin{aligned}
& T_{v_{12} \leftarrow(1 s)_{23}}^{\text {ortho }}=-\frac{Z_{2} Z_{3}}{\sqrt{2} \pi^{3}}\left[\frac{m_{1}}{m_{1}+m_{2}}\right]^{3} \sum_{n_{\lambda}, l_{\lambda}}\left(2 l_{\lambda}+1\right) \int d p d \theta_{p} \sin \theta_{p} \frac{p^{2}}{\left|\mathbf{p}+\mathbf{q}_{2}\right|^{2}} R_{n_{\lambda} l_{\lambda}}(p) I_{\bar{v} \lambda}\left(\left|\mathbf{p}+\mathbf{q}_{2}\right|\right)_{l_{\bar{v}}=0} \\
& \times P_{l_{\lambda}}\left(\frac{\mathbf{p} \cdot\left(\mathbf{p}+\mathbf{q}_{2}\right)}{p\left|\mathbf{p}+\mathbf{q}_{2}\right|}\right) \frac{A}{\left(A^{2}-B^{2}\right)^{3 / 2}} \text { for } l_{\bar{v}}=0
\end{aligned}
$$

\section{DIFFERENTIAL AND TOTAL CROSS SECTIONS}

The integral appearing in Eq. (5.10) is evaluated easily and rapidly by two-dimensional numerical integration. When the electron mass is neglected in relation to the nuclear mass, this integral can be evaluated analytically for each value of $n_{\lambda}$ and $l_{\lambda}$, the intermediate-state quantum numbers. We have done this for all states up to $n_{\lambda}=3$ as a check on the result of numerical integration.

In the numerical results that we quote, only the $1 s, 2 s$, and $2 p$ intermediate states have been retained in the sum in Eq. (5.10). This a very good approximation at all energies for which we present differential cross section $(25,60$, ad $125 \mathrm{keV}$ ) in Fig. 4 but is somewhat suspect at the lower end of the energy scale $(E<10 \mathrm{keV})$ of Fig. 5 where we

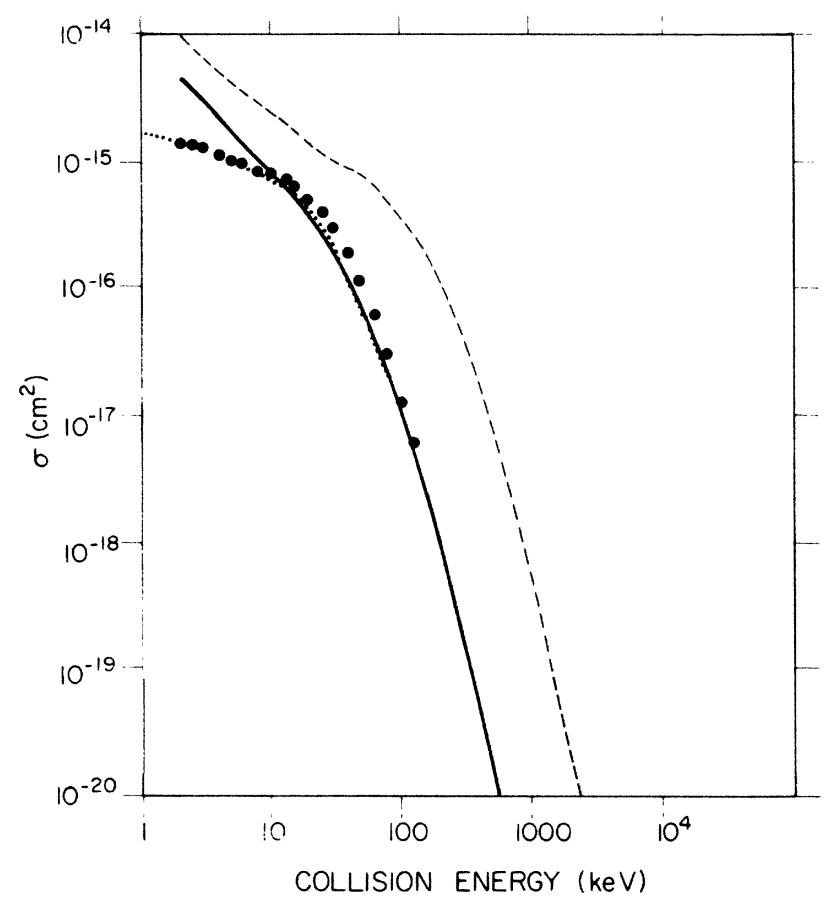

FIG. 5. Total cross section for resonant charge transfer, $p+\mathbf{H}(1 s) \rightarrow \mathrm{H}(1 s)+p$, as a function of laboratory collision energy. The experimental points are from McClure (Ref. 19), the dotted curve is from the two-state atomic expansion calculation of McCarroll (Ref. 20), and the solid curve is the Fock-Tani result with orthogonality corrections due to $1 s, 2 s$, and $2 p$ intermediate states. The experimental cross section in this case is also for capture into all bound states. The dashed curve gives the Fock-Tani cross section for $d+\mathbf{H}(1 s) \rightarrow \mathrm{D}(1 s)+p$. present the total cross section. However, the error is estimated to be no more than $20 \%$, and at these low energies, a first-order approximation is inadequate anyway.

We compare our calculated differential cross section with the experimental results (Martin et al. ${ }^{17}$ ) in Fig. 4 and also with the two-state atomic expansion (TSAE) results quoted by Martin et al. The TSAE approximation was picked out of a number of theoretical results because it seems to give the best fit to differential and total cross sections over a large range of angles and energies. It is satisfying to see that our calculation agrees very well with the TSAE and experimental results over angular range of $1 \mathrm{mrad}$ about the forward direction and over a comparable range at 60 and $125 \mathrm{keV}$ also. The discrepancy at larger angles is no doubt due to the neglect of higherorder corrections and we expect much of it to disappear upon the inclusion of second-order correction. In fact, even the minima in the first-order differential cross section at the energies we have considered are spurious. ${ }^{18}$

We also compare the Fock-Tani total cross section with the experimental results of McClure ${ }^{19}$ and the TSAE calculation of $\mathrm{McCarroll}^{20}$ in Fig. 5. Once again we find that our calculation agrees very well with both these results for $E>10 \mathrm{keV}$; in fact, for $E>60 \mathrm{keV}$, our results are identical with those of McCarroll. The discrepancy at low energies is to be expected of a first-order approximation. In the same figure, we also show the Fock-Tani total cross section for $d+\mathrm{H}(1 s) \rightarrow \mathrm{D}(1 s)+p$.

We close our discussion of the numerical results by remarking that the two-state atomic expansion was introduced by Bates ${ }^{8}$ with the express purpose of correcting for the lack of orthogonality of the initial- and final-state wave functions. This, along with other orthogonality corrections, is contained in the Fock-Tani approximation, too. Close agreement between the results of these approximations is, then, just as it should be.

\section{SUMMARY AND CONCLUSION}

In this paper we have developed a unitary transformation of the second-quantized Hamiltonian of a threeparticle system which breaks the overall interaction into its component parts corresponding to permissible physical processes and have applied it to the problem of resonant charge transfer, $p+\mathrm{H}(1 s) \rightarrow \mathrm{H}(1 s)+p$. In this scheme, zeroth-order wave functions for initial and final states are mutually orthogonal, thus satisfying the same orthogonality relation as the exact scattering states. As a consequence of this, an orthogonality correction to the usual first-order reaction amplitude appears and the internuclear potential makes a negligible contribution of order 
$m_{e} / m_{p}$ to the $T$-matrix element. This result is consistent with the physical result that the internuclear potential cannot significantly affect the passage of a light, charged particle from one nucleus to another. We have computed differential and total cross sections over a range of angles and energies and compared our results with experiment and with the best available theoretical results based on a two-state atomic expansion. Except at angles larger than $1 \mathrm{mrad}$ and energies less than $10 \mathrm{keV}$, we find very good agreement. The discrepancy is attributed to the omission of second- and higher-order perturbative effects. The agreement with TSAE results is a reflection of the fact that this method also corrects for the lack of orthogonality of the zeroth-order initial and final states in Fock representation. The agreement with experiment underlines the importance of proper orthogonality of approximate scattering states, a fact recognized long back by Bates. ${ }^{8}$

An obvious extension of the present work is to include second-order corrections and thus attempt to remove the remaining discrepancy between our results and the experiment. Such an extension is currently being investigated. We are also contemplating application of Fock-Tani formalism to other problems of atomic scattering, particularly ionization of the hydrogen atom by electron impact.

\section{ACKNOWLEDGMENT}

This research was supported in part by the U.S. Office of Naval Research.

\section{APPENDIX A: FOCK-TANI TRANSFORMATION FOR THE THREE-PARTICLE SYSTEM}

We have evaluated the Fock-Tani Hamiltonian (3.4a) by a diagrammatic technique. ${ }^{21}$ The interaction is now broken up into various parts corresponding to the possible physical processes for this system:

$$
\begin{aligned}
& \hat{H}_{\mathrm{FT} 1}=\sum_{\alpha, \mu_{\alpha}} \epsilon_{\mu_{\alpha}}^{\alpha}\left(\hat{b}_{\mu_{\alpha}}^{\alpha}\right)^{\dagger} \hat{b}_{\mu_{\alpha}}^{\alpha}+\sum_{i} \int d \mathbf{r}_{i} \hat{a}_{i}^{\dagger}\left(\mathbf{r}_{i}\right) T_{i}\left(\mathbf{r}_{i}\right) \hat{a}_{i}\left(\mathbf{r}_{i}\right) \\
& +\sum_{\substack{i, j \\
(j>i)}} \int d \mathbf{r}_{i} d \mathbf{r}_{j} d \mathbf{r}_{i}^{\prime} d \mathbf{r}_{j}^{\prime} \hat{a}_{i}^{\dagger}\left(\mathbf{r}_{i}\right) \hat{a}_{j}^{\dagger}\left(\mathbf{r}_{j}\right)\left\langle\mathbf{r}_{i} \mathbf{r}_{j}|V| \mathbf{r}_{i}^{\prime} \mathbf{r}_{j}^{\prime}\right\rangle \widehat{a}_{j}\left(\mathbf{r}_{j}^{\prime}\right) \widehat{a}_{i}\left(\mathbf{r}_{i}^{\prime}\right) \\
& +\sum_{\alpha, \mu_{\alpha}, v_{\alpha}} \int d \mathbf{r}_{\bar{\alpha}} d \mathbf{r}_{\bar{\alpha}}^{\prime}\left(\hat{b}_{\mu_{\alpha}}^{\alpha}\right)^{\dagger} \widehat{a}_{\bar{\alpha}}^{\dagger}\left(\mathbf{r}_{\bar{\alpha}}\right)\left\langle\mu_{\alpha} \mathbf{r}_{\bar{\alpha}}|V| v_{\alpha} \mathbf{r}_{\bar{\alpha}}^{\prime}\right\rangle \widehat{a}_{\bar{a}}\left(\mathbf{r}_{\bar{\alpha}}^{\prime}\right) \hat{b}_{v_{\alpha}}^{\alpha} \\
& +\left[\sum_{\alpha, \mu_{\alpha}} \int d \mathbf{r}_{\bar{\alpha}} d \mathbf{r}_{i}^{\prime} d \mathbf{r}_{j}^{\prime} d \mathbf{r}_{\bar{a}}^{\prime}\left(\hat{b}_{\mu_{\alpha}}^{\alpha}\right)^{\dagger} \widehat{a}_{\bar{a}}^{\dagger}\left(\mathbf{r}_{\bar{\alpha}}\right)\left\langle\mu_{\alpha} \mathbf{r}_{\bar{\alpha}}|V| \mathbf{r}_{i}^{\prime} \mathbf{r}_{j}^{\prime} \mathbf{r}_{\bar{\alpha}}^{\prime}\right) \widehat{a}_{\bar{\alpha}}\left(\mathbf{r}_{\bar{a}}^{\prime}\right) \widehat{a}_{j}\left(\mathbf{r}_{j}^{\prime}\right) \widehat{a}_{i}\left(\mathbf{r}_{i}^{\prime}\right)+\text { H.c. }\right] \\
& +\left[\sum_{\mu_{12}, \mu_{23}} \int d \mathbf{r}_{3} d \mathbf{r}_{1}\left(\hat{b}_{\mu_{12}}^{(12)}\right)^{\dagger} \hat{a}_{3}^{\dagger}\left(\mathbf{r}_{3}\right)\left\langle\mu_{12} \mathbf{r}_{3}|V| \mu_{23} \mathbf{r}_{1}\right) \widehat{a}_{1}\left(\mathbf{r}_{1}\right) \hat{b}_{\mu_{23}}^{(23)}+\text { H.c. }\right) \\
& +\int\left\{\prod_{i} d \mathbf{r}_{i} d \mathbf{r}_{i}^{\prime}\right] \widehat{a}_{1}^{\dagger}\left(\mathbf{r}_{1}\right) \widehat{a}_{2}^{\dagger}\left(\mathbf{r}_{2}\right) \widehat{a}_{3}^{\dagger}\left(\mathbf{r}_{3}\right)\left\langle\mathbf{r}_{1} \mathbf{r}_{2} \mathbf{r}_{3}|V| \mathbf{r}_{1}^{\prime} \mathbf{r}_{2}^{\prime} \mathbf{r}_{3}^{\prime}\right\rangle \widehat{a}_{3}\left(\mathbf{r}_{3}^{\prime}\right) \widehat{a}_{2}\left(\mathbf{r}_{2}^{\prime}\right) \widehat{a}_{1}\left(\mathbf{r}_{1}^{\prime}\right) .
\end{aligned}
$$

In our notation $\alpha \equiv(i j)$ labels the pair $(i, j)$ and $\bar{\alpha}$ labels the remaining particle. The sum over $\alpha$ ranges from $\alpha_{1}=(12)$ to $\alpha_{2}=(23)$ and correspondingly $\bar{\alpha}_{1}=3$ and $\bar{\alpha}_{2}=1$. Indices $i$ and $j$, when summed, range from 1 to 3 but are otherwise defined by associated $\alpha$. All interactions are written formally as brackets, $\langle||\rangle$, and the symbol H.c. denotes Hermitian conjugation.

The first two terms in (A1) constitute the unperturbed Hamiltonian $\hat{H}_{0}$ for our calculation. The eigenstates of $\hat{H}_{0}$ include plane waves arising from the second term, impinging on bound states, arising from the first term. Explicit expressions for the interactions appearing in (A1) and their physical significance are as follows.

(a) Two-particle scattering. The interaction for two-particle scattering is given by

$$
\left\langle\mathbf{r}_{i} \mathbf{r}_{j}|V| \mathbf{r}_{i}^{\prime} \mathbf{r}_{j}^{\prime}\right\rangle=V_{i j}\left(\mathbf{r}_{i}, \mathbf{r}_{j}\right) \delta\left(\mathbf{r}_{i}-\mathbf{r}_{i}^{\prime}\right) \delta\left(\mathbf{r}_{j}-\mathbf{r}_{j}^{\prime}\right)-\left(1-\delta_{i j, 13}\right) \sum_{\mu_{\alpha}} \epsilon_{\mu_{\alpha}}^{\alpha} \Delta_{\mu_{\alpha}}^{\alpha}\left(\mathbf{r}_{i}, \mathbf{r}_{j} ; \mathbf{r}_{i}^{\prime}, \mathbf{r}_{j}^{\prime}\right) \text {. }
$$

The second term in (A2) expresses the subtraction of the bound-state part of the interaction from the total interaction to give the remaining "scattering" interaction. (In this equation $\Delta_{\mu_{\alpha}}^{\alpha}\left(\mathbf{r}_{i}, \mathbf{r}_{j} ; \mathbf{r}_{i}^{\prime}, \mathbf{r}_{j}^{\prime}\right)=\psi_{\mu_{\alpha}}^{\alpha}\left(\mathbf{r}_{i}, \mathbf{r}_{j}\right)\left[\psi_{\mu_{\alpha}}^{\alpha}\left(\mathbf{r}_{i}^{\prime}, \mathbf{r}_{j}^{\prime}\right)\right]^{*}$.) Note that it is zero for the pair (13) which is assumed not to form a bound state.

(b) Nonreactive scattering of $\bar{\alpha}$ from the bound states of $\alpha$. Due to the $\left(\alpha_{1} \rightleftarrows \alpha_{2}\right)$ asymmetry of the transformation in (3.4a), this interaction is not symmetric for the two arrangement channels:

$$
\left\langle\mu_{12} \mathbf{r}_{3}|V| \boldsymbol{v}_{12} \mathbf{r}_{3}^{\prime}\right\rangle=\int d \mathbf{r}_{1} d \mathbf{r}_{2}\left[\psi_{\mu_{12}}^{(12)}\left(\mathbf{r}_{1}, \mathbf{r}_{2}\right)\right] *\left[V_{13}\left(\mathbf{r}_{1}, \mathbf{r}_{3}\right)+V_{23}\left(\mathbf{r}_{2}, \mathbf{r}_{3}\right)\right] \psi_{v_{12}}\left(\mathbf{r}_{1}, \mathbf{r}_{2}\right) \delta\left(\mathbf{r}_{3}-\mathbf{r}_{3}^{\prime}\right)
$$




$$
\begin{aligned}
& \left\langle\mu_{23} \mathbf{r}_{1}|V| v_{23} \mathbf{r}_{1}^{\prime}\right\rangle=\int d \mathbf{r}_{2} d \mathbf{r}_{3}\left[\psi_{\mu_{23}}^{(23)}\left(\mathbf{r}_{2}, \mathbf{r}_{3}\right)\right]^{*}\left[V_{12}\left(\mathbf{r}_{1}, \mathbf{r}_{2}\right)+V_{13}\left(\mathbf{r}_{1}, \mathbf{r}_{3}\right)\right] \psi_{v_{23}}^{(23)}\left(\mathbf{r}_{2}, \mathbf{r}_{3}\right) \delta\left(\mathbf{r}_{1}-\mathbf{r}_{1}^{\prime}\right) \\
& -\sum_{\lambda_{12}} \int d \mathbf{r}_{2} d \mathbf{r}_{3} d \mathbf{r}_{2}^{\prime}\left[\psi_{\mu_{23}}^{(23)}\left(\mathbf{r}_{2}, \mathbf{r}_{3}\right)\right]^{*}\left[V_{13}\left(\mathbf{r}_{1}, \mathbf{r}_{3}\right)+V_{13}\left(\mathbf{r}_{1}^{\prime}, \mathbf{r}_{3}\right)+V_{23}\left(\mathbf{r}_{2}, \mathbf{r}_{3}\right)+V_{23}\left(\mathbf{r}_{2}^{\prime}, \mathbf{r}_{3}\right)+\epsilon_{\lambda_{12}}^{(12)}\right] \\
& \quad \times \Delta_{\lambda_{12}}^{(12)}\left(\mathbf{r}_{1}, \mathbf{r}_{2} ; \mathbf{r}_{1}^{\prime}, \mathbf{r}_{2}^{\prime}\right) \psi_{v_{23}}^{(23)}\left(\mathbf{r}_{2}^{\prime}, \mathbf{r}_{3}\right) \\
& +\int d \mathbf{r}_{2} d \mathbf{r}_{3} d \mathbf{r}_{2}^{\prime} d \mathbf{r}_{1}^{\prime \prime} d \mathbf{r}_{2}^{\prime \prime}\left[\psi_{\mu_{23}}^{(23)}\left(\mathbf{r}_{2}, \mathbf{r}_{3}\right)\right]^{*} \Delta^{(12)}\left(\mathbf{r}_{1}, \mathbf{r}_{2} ; \mathbf{r}_{1}^{\prime \prime}, \mathbf{r}_{2}^{\prime \prime}\right) \\
& \quad \times\left[V_{13}\left(\mathbf{r}_{1}^{\prime \prime}, \mathbf{r}_{3}\right)+V_{23}\left(\mathbf{r}_{2}^{\prime \prime}, \mathbf{r}_{3}\right)\right] \Delta^{(12)}\left(\mathbf{r}_{1}^{\prime \prime}, \mathbf{r}_{2}^{\prime \prime} ; \mathbf{r}_{1}^{\prime}, \mathbf{r}_{2}^{\prime}\right) \psi_{\mu_{23}}^{(23)}\left(\mathbf{r}_{2}^{\prime}, \mathbf{r}_{3}\right)
\end{aligned}
$$

(c) Formation and breakup of bound states. In this case also the interaction is different for pairs (12) and (23):

$$
\begin{aligned}
\left\langle\mu_{12} \mathbf{r}_{3}|V| \mathbf{r}_{1}^{\prime} \mathbf{r}_{2}^{\prime} \mathbf{r}_{3}^{\prime}\right\rangle= & \int d \mathbf{r}_{1} d \mathbf{r}_{2}\left[\psi_{\mu_{12}}^{(12)}\left(\mathbf{r}_{1}, \mathbf{r}_{2}\right)\right]^{*}\left[V_{13}\left(\mathbf{r}_{1}, \mathbf{r}_{3}\right)+V_{23}\left(\mathbf{r}_{2}, \mathbf{r}_{3}\right)\right] \\
& \times\left[\delta\left(\mathbf{r}_{1}-\mathbf{r}_{1}^{\prime}\right) \delta\left(\mathbf{r}_{2}-\mathbf{r}_{2}^{\prime}\right)-\Delta^{(12)}\left(\mathbf{r}_{1}, \mathbf{r}_{2} ; \mathbf{r}_{1}^{\prime}, \mathbf{r}_{2}^{\prime}\right)\right] \delta\left(\mathbf{r}_{3}-\mathbf{r}_{3}^{\prime}\right) \\
+ & \int d \mathbf{r}_{2} d \mathbf{r}_{1}^{\prime \prime} d \mathbf{r}_{2}^{\prime \prime}\left[\psi_{\mu_{12}}^{(12)}\left(\mathbf{r}_{1}^{\prime \prime}, \mathbf{r}_{2}^{\prime \prime}\right)\right]^{*}\left[V_{13}\left(\mathbf{r}_{1}^{\prime \prime}, \mathbf{r}_{3}\right)+V_{23}\left(\mathbf{r}_{2}^{\prime \prime}, \mathbf{r}_{3}\right)\right] \\
& \times\left[\Delta^{(12)}\left(\mathbf{r}_{1}^{\prime \prime}, \mathbf{r}_{2}^{\prime \prime} ; \mathbf{r}_{1}^{\prime}, \mathbf{r}_{2}\right)-\delta\left(\mathbf{r}_{1}^{\prime}-\mathbf{r}_{1}^{\prime \prime}\right) \delta\left(\mathbf{r}_{2}^{\prime}-\mathbf{r}_{2}^{\prime \prime}\right)\right] \Delta^{(23)}\left(\mathbf{r}_{2}, \mathbf{r}_{3} ; \mathbf{r}_{2}^{\prime}, \mathbf{r}_{3}^{\prime}\right)
\end{aligned}
$$

and

$$
\begin{aligned}
\left\langle\mu_{23} \mathbf{r}_{1}|V| \mathbf{r}_{2}^{\prime} \mathbf{r}_{3}^{\prime} \mathbf{r}_{1}^{\prime}\right\rangle= & \int d \mathbf{r}_{2} d \mathbf{r}_{3}\left[\psi_{\mu_{23}}^{(23)}\left(\mathbf{r}_{2}, \mathbf{r}_{3}\right)\right]^{*}\left[V_{12}\left(\mathbf{r}_{1}, \mathbf{r}_{2}\right)+V_{13}\left(\mathbf{r}_{1}, \mathbf{r}_{3}\right)\right] \\
& \times\left[\delta\left(\mathbf{r}_{2}-\mathbf{r}_{2}^{\prime}\right) \delta\left(\mathbf{r}_{3}-\mathbf{r}_{3}^{\prime}\right)-\Delta^{(23)}\left(\mathbf{r}_{2}, \mathbf{r}_{3} ; \mathbf{r}_{2}^{\prime}, \mathbf{r}_{3}^{\prime}\right)\right] \delta\left(\mathbf{r}_{1}-\mathbf{r}_{1}^{\prime}\right) \\
& -\sum_{v_{12}} \int d \mathbf{r}_{2}\left[\psi_{\mu_{23}}^{(23)}\left(\mathbf{r}_{2}, \mathbf{r}_{3}^{\prime}\right)\right]^{*}\left[V_{13}\left(\mathbf{r}_{1}, \mathbf{r}_{3}^{\prime}\right)+V_{23}\left(\mathbf{r}_{2}, \mathbf{r}_{3}^{\prime}\right)+V_{13}\left(\mathbf{r}_{1}^{\prime}, \mathbf{r}_{3}^{\prime}\right)+V_{23}\left(\mathbf{r}_{2}^{\prime}, \mathbf{r}_{3}^{\prime}\right)+\epsilon_{v_{12}}^{(12)}\right] \Delta_{v_{12}}^{(12)}\left(\mathbf{r}_{1}, \mathbf{r}_{2} ; \mathbf{r}_{1}^{\prime}, \mathbf{r}_{2}^{\prime}\right) \\
+ & \int d \mathbf{r}_{2} d \mathbf{r}_{1}^{\prime \prime} d \mathbf{r}_{2}^{\prime \prime}\left[\psi_{\mu_{23}}^{(23)}\left(\mathbf{r}_{2}, \mathbf{r}_{3}^{\prime}\right)\right]^{*} \Delta^{(12)}\left(\mathbf{r}_{1}, \mathbf{r}_{2} ; \mathbf{r}_{1}^{\prime \prime}, \mathbf{r}_{2}^{\prime \prime}\right)\left[V_{13}\left(\mathbf{r}_{1}^{\prime \prime}, \mathbf{r}_{3}^{\prime}\right)+V_{23}\left(\mathbf{r}_{2}^{\prime \prime}, \mathbf{r}_{3}^{\prime}\right)\right] \Delta^{(12)}\left(\mathbf{r}_{1}^{\prime \prime}, \mathbf{r}_{2}^{\prime \prime} ; \mathbf{r}_{1}^{\prime}, \mathbf{r}_{2}^{\prime}\right)
\end{aligned}
$$

(d) Rearrangement interaction. The interaction for breakup of (23) and formation of (12) is given by

$$
\begin{aligned}
\left\langle\mu_{12} \mathbf{r}_{3}|V| \mu_{23} \mathbf{r}_{1}\right\rangle=\int & d \mathbf{r}_{2} d \mathbf{r}_{1}^{\prime} d \mathbf{r}_{2}^{\prime}\left[\psi_{\mu_{12}}^{(12)}\left(\mathbf{r}_{1}^{\prime}, \mathbf{r}_{2}^{\prime}\right)\right]^{*}\left[V_{13}\left(\mathbf{r}_{1}^{\prime}, \mathbf{r}_{3}^{\prime}\right)+V_{23}\left(\mathbf{r}_{2}^{\prime}, \mathbf{r}_{3}^{\prime}\right)\right] \\
& \times\left[\delta\left(\mathbf{r}_{1}-\mathbf{r}_{1}^{\prime}\right) \delta\left(\mathbf{r}_{2}-\mathbf{r}_{2}^{\prime}\right)-\Delta^{(12)}\left(\mathbf{r}_{1}^{\prime}, \mathbf{r}_{2}^{\prime} ; \mathbf{r}_{1}, \mathbf{r}_{2}\right)\right] \psi_{\mu_{23}}^{(23)}\left(\mathbf{r}_{2}, \mathbf{r}_{3}\right) .
\end{aligned}
$$

The interaction for the reverse process is obtained by complex conjugation.

(e) Three-particle scattering. The interaction for three-particle scattering is given by

$$
\begin{aligned}
\left\langle\mathbf{r}_{1} \mathbf{r}_{2} \mathbf{r}_{3}|V| \mathbf{r}_{1}^{\prime} \mathbf{r}_{2}^{\prime} \mathbf{r}_{3}^{\prime}\right\rangle= & -\sum_{\alpha} \Delta^{\alpha}\left(\mathbf{r}_{i}, \mathbf{r}_{j} ; \mathbf{r}_{i}^{\prime}, \mathbf{r}_{j}^{\prime}\right)\left[V_{i \bar{\alpha}}\left(\mathbf{r}_{i}, \mathbf{r}_{\bar{\alpha}}\right)+V_{j \bar{\alpha}}\left(\mathbf{r}_{j}, \mathbf{r}_{\bar{\alpha}}\right)+V_{i \alpha}\left(\mathbf{r}_{i}^{\prime}, \mathbf{r}_{\bar{\alpha}}^{\prime}\right)+V_{j \bar{\alpha}}\left(\mathbf{r}_{j}^{\prime}, \mathbf{r}_{\bar{\alpha}}^{\prime}\right)\right] \delta\left(\mathbf{r}_{\bar{\alpha}}-\mathbf{r}_{\bar{\alpha}}^{\prime}\right) \\
& +\sum_{\alpha} \int d \mathbf{r}_{i}^{\prime \prime} d \mathbf{r}_{j}^{\prime \prime} \Delta^{\alpha}\left(\mathbf{r}_{i}, \mathbf{r}_{j} ; \mathbf{r}_{i}^{\prime \prime}, \mathbf{r}_{j}^{\prime \prime}\right)\left[V_{i \bar{\alpha}}\left(\mathbf{r}_{i}^{\prime \prime}, \mathbf{r}_{\bar{\alpha}}\right)+V_{j \bar{\alpha}}\left(\mathbf{r}_{j}^{\prime \prime}, \mathbf{r}_{\bar{\alpha}}\right)\right] \Delta^{\alpha}\left(\mathbf{r}_{i}^{\prime \prime}, \mathbf{r}_{j}^{\prime \prime} ; \mathbf{r}_{i}^{\prime}, \mathbf{r}_{j}^{\prime}\right) \delta\left(\mathbf{r}_{\bar{\alpha}}-\mathbf{r}_{\bar{\alpha}}^{\prime}\right) \\
& +\sum_{\mu_{12}} \int d \mathbf{r}_{2}^{\prime \prime} d \mathbf{r}_{3}^{\prime \prime}\left[V_{13}\left(\mathbf{r}_{1}, \mathbf{r}_{3}^{\prime \prime}\right)+V_{23}\left(\mathbf{r}_{2}^{\prime \prime}, \mathbf{r}_{3}^{\prime \prime}\right)+V_{13}\left(\mathbf{r}_{1}^{\prime}, \mathbf{r}_{3}^{\prime \prime}\right)+V_{23}\left(\mathbf{r}_{2}^{\prime}, \mathbf{r}_{3}^{\prime \prime}\right)+\epsilon_{\mu_{12}}^{(12)}\right] \\
& \times\left[\delta\left(\mathbf{r}_{3}-\mathbf{r}_{3}^{\prime \prime}\right) \Delta_{\mu_{12}}^{(12)}\left(\mathbf{r}_{1}, \mathbf{r}_{2} ; \mathbf{r}_{1}^{\prime}, \mathbf{r}_{2}^{\prime \prime}\right) \Delta^{\alpha_{2}}\left(\mathbf{r}_{2}^{\prime \prime}, \mathbf{r}_{3}^{\prime \prime} ; \mathbf{r}_{2}^{\prime}, \mathbf{r}_{3}^{\prime}\right)+\delta\left(\mathbf{r}_{3}^{\prime}-\mathbf{r}_{3}^{\prime \prime}\right) \Delta^{\alpha_{2}}\left(\mathbf{r}_{2}, \mathbf{r}_{3} ; \mathbf{r}_{2}^{\prime \prime}, \mathbf{r}_{3}^{\prime \prime}\right) \Delta_{\mu_{12}}^{(12)}\left(\mathbf{r}_{1}, \mathbf{r}_{2}^{\prime \prime} ; \mathbf{r}_{1}^{\prime}, \mathbf{r}_{2}^{\prime}\right)\right] \\
& -\int d \mathbf{r}_{2}^{\prime \prime} d \mathbf{r}_{1}^{\prime \prime \prime} d \mathbf{r}_{2}^{\prime \prime \prime} \Delta^{\alpha_{1}}\left(\mathbf{r}_{1}, \mathbf{r}_{2} ; \mathbf{r}_{1}^{\prime \prime \prime} \mathbf{r}_{2}^{\prime \prime \prime}\right)\left[V_{13}\left(\mathbf{r}_{1}^{\prime \prime \prime}, \mathbf{r}_{3}\right)+V_{23}\left(\mathbf{r}_{2}^{\prime \prime \prime}, \mathbf{r}_{3}\right)\right] \Delta^{\alpha_{1}}\left(\mathbf{r}_{1}^{\prime \prime \prime}, \mathbf{r}_{2}^{\prime \prime \prime} ; \mathbf{r}_{1}^{\prime}, \mathbf{r}_{2}^{\prime \prime}\right) \Delta^{\alpha_{2}}\left(\mathbf{r}_{2}^{\prime \prime}, \mathbf{r}_{3} ; \mathbf{r}_{2}^{\prime}, \mathbf{r}_{3}^{\prime}\right) \\
& -\int d \mathbf{r}_{2}^{\prime \prime} d \mathbf{r}_{1}^{\prime \prime \prime} d \mathbf{r}_{2}^{\prime \prime \prime} \Delta^{\alpha_{2}}\left(\mathbf{r}_{2}, \mathbf{r}_{3} ; \mathbf{r}_{2}^{\prime \prime}, \mathbf{r}_{3}^{\prime}\right) \Delta^{\alpha_{1}}\left(\mathbf{r}_{1}, \mathbf{r}_{2}^{\prime \prime} ; \mathbf{r}_{1}^{\prime \prime \prime}, \mathbf{r}_{2}^{\prime \prime \prime}\right)\left[V_{13}\left(\mathbf{r}_{1}^{\prime \prime \prime}, \mathbf{r}_{3}^{\prime}\right)+V_{23}\left(\mathbf{r}_{2}^{\prime \prime \prime}, \mathbf{r}_{3}^{\prime}\right)\right] \Delta^{\alpha_{1}}\left(\mathbf{r}_{1}^{\prime \prime \prime}, \mathbf{r}_{2}^{\prime \prime \prime} ; \mathbf{r}_{1}^{\prime}, \mathbf{r}_{2}^{\prime}\right) \\
& -\sum_{\mu_{12} \int d \mathbf{r}_{2}^{\prime \prime} d \mathbf{r}_{3}^{\prime \prime} d \mathbf{r}_{2}^{\prime \prime \prime} \Delta^{\alpha_{2}}\left(\mathbf{r}_{2}, \mathbf{r}_{3} ; \mathbf{r}_{2}^{\prime \prime}, \mathbf{r}_{3}^{\prime \prime}\right)} \\
& \times\left[V_{13}\left(\mathbf{r}_{1}, \mathbf{r}_{3}^{\prime \prime}\right)+V_{23}\left(\mathbf{r}_{2}, \mathbf{r}_{3}^{\prime \prime}\right)+V_{13}\left(\mathbf{r}_{1}^{\prime}, \mathbf{r}_{3}^{\prime \prime}\right)+V_{23}\left(\mathbf{r}_{2}^{\prime \prime \prime}, \mathbf{r}_{3}^{\prime \prime}\right)+\epsilon_{\mu_{12}}^{(12)}\right]
\end{aligned}
$$




$$
\begin{aligned}
& \quad \times \Delta_{\mu_{12}}^{(12)}\left(\mathbf{r}_{1}, \mathbf{r}_{2} ; \mathbf{r}_{1}^{\prime}, \mathbf{r}_{2}^{\prime \prime \prime}\right) \Delta^{\alpha_{2}}\left(\mathbf{r}_{2}^{\prime \prime \prime}, \mathbf{r}_{3}^{\prime \prime} ; \mathbf{r}_{2}^{\prime}, \mathbf{r}_{3}^{\prime}\right) \\
& +\int d \mathbf{r}_{2}^{\prime \prime} d \mathbf{r}_{3}^{\prime \prime} d \mathbf{r}_{1}^{\prime \prime} d \mathbf{r}_{2}^{\prime \prime \prime} d \mathbf{r}_{2}^{\prime \prime \prime \prime} \Delta^{\alpha_{2}}\left(\mathbf{r}_{2}, \mathbf{r}_{3} ; \mathbf{r}_{2}^{\prime \prime}, \mathbf{r}_{3}^{\prime \prime}\right) \Delta^{\alpha_{1}}\left(\mathbf{r}_{1}, \mathbf{r}_{2}^{\prime \prime} ; \mathbf{r}_{1}^{\prime \prime \prime}, \mathbf{r}_{2}^{\prime \prime \prime}\right) \\
& \times\left[V_{13}\left(\mathbf{r}_{1}^{\prime \prime \prime}, \mathbf{r}_{3}^{\prime \prime}\right)+V_{23}\left(\mathbf{r}_{2}^{\prime \prime \prime}, \mathbf{r}_{3}^{\prime \prime}\right)\right] \Delta^{\alpha_{2}}\left(\mathbf{r}_{2}^{\prime \prime \prime}, \mathbf{r}_{3}^{\prime \prime} ; \mathbf{r}_{2}^{\prime}, \mathbf{r}_{3}^{\prime}\right)
\end{aligned}
$$

\section{APPENDIX B: ASSORTED ANALYTICAL INTEGRALS IN THE EVALUATION OF THE ORTHOGONALITY CORRECTION}

For specific final and intermediate states, the integral in (5.7) is evaluated by a spherical harmonic expansion of the plane wave. The result for the $1 s$ final state and the $1 s$, $2 s$, and $2 p$ intermediate states is as follows:

$$
\begin{aligned}
& I_{1 s, 1 s}\left(\mathbf{p}+\mathbf{q}_{2}\right)=\frac{32 \sqrt{\pi}}{\left(4+\left|\mathbf{p}+\mathbf{q}_{2}\right|^{2}\right)^{2}} Y_{00}\left(\Omega_{\mathrm{p}+\mathrm{q}_{2}}\right), \\
& I_{1 s, 2 s}\left(\mathbf{p}+\mathbf{q}_{2}\right)=\frac{512 \sqrt{2 \pi}\left|\mathbf{p}+\mathbf{q}_{2}\right|^{2}}{\left(q+4\left|\mathbf{p}+\mathbf{q}_{2}\right|^{2}\right)^{3}} Y_{00}\left(\Omega_{\mathrm{p}+\mathbf{q}_{2}}\right)
\end{aligned}
$$

and

$$
I_{1 s, 2 p m}\left(p+q_{2}\right)=-i \frac{256 \sqrt{6 \pi}\left|p+q_{2}\right|}{\left(9+4\left|p+q_{2}\right|^{2}\right)^{3}} Y_{1 m}\left(\Omega_{p+q_{2}}\right) .
$$

In Eq. (5.6) the integral of the initial-state wave function over the coordinate $\phi_{p}$, for the $1 s$ initial state $(\bar{\mu}=1 s)$, is explicitly written as

$$
\begin{aligned}
I \equiv & \int_{0}^{2 \pi} d \phi_{p} \Phi_{\mu}\left(\frac{m_{1}+m_{2}}{m_{1}}\left(\mathbf{p}+\mathbf{m} \mathbf{k}_{i}\right)\right] \\
= & \frac{2 \sqrt{2}}{\pi}\left[\frac{m_{1}}{m_{1}+m_{2}}\right]^{4} \\
& \times \int_{0}^{2 \pi} d \phi_{p}\left[\left(\frac{m_{1}}{m_{1}+m_{2}}\right]^{2}+p^{2}+2 \mathbf{m} \mathbf{p} \cdot \mathbf{k}_{i}+\mathbf{m}^{2} k_{i}^{2}\right]^{-2} .
\end{aligned}
$$

The only $\phi_{p}$ dependence of the integrand comes from the $\mathbf{p} \cdot \mathbf{k}_{i}$ term which is expanded in terms of trigonometric functions of angles $\phi_{p}, \theta_{p}$, and $\theta_{k_{i}}$ with reference to Fig. 3. Noting that the integrand is of the form $\left(A+B \cos \phi_{p}\right)^{-2}$, where

$$
A=\left(\frac{m_{1}}{m_{1}+m_{2}}\right)^{2}+p^{2}+2 m p k_{i} \cos \theta_{p} \cos \theta_{k_{i}}+m^{2} k_{i}^{2}
$$

and

$$
B=2 \mathrm{~m} p k_{i} \sin \theta_{p} \sin \theta_{k_{i}},
$$

the integral is evaluated analytically ${ }^{22}$ to give

$$
I=4 \sqrt{2}\left(\frac{m_{1}}{m_{1}+m_{2}}\right)^{4} \frac{A}{\left(A^{2}-B^{2}\right)^{3 / 2}} .
$$

The angle $\theta_{k_{i}}$ in Eq. (B3) is related to the scattering angle $\theta_{i f}$ once again with reference to Fig. 3 .
${ }^{1}$ E. Ficocelli Varrachio and M. D. Girardeau, J. Phys. B 16, 1097 (1983).

${ }^{2}$ E. Ficocelli Varrachio, J. Phys. B 17, L311 (1984).

3J. R. Oppenheimer, Phys. Rev. 31, 349 (1928).

${ }^{4}$ H. C. Brinkman and H. A. Kramers, Proc. K. Akad. Wet. Amst. 33, 973 (1930).

5Dz. Belkic, R. Gayet, and A. Salin, Phys. Rep. 56, 279 (1979).

6J. D. Jackson and H. Schiff, Phys. Rev. 89, 359 (1953).

${ }^{7}$ D. R. Bates and A. Dalgarno, Proc. R. Soc. London, Ser. A 65, 919 (1952).

${ }^{8}$ D. R. Bates, Proc. R. Soc. London, Ser. A 247, 294 (1958).

${ }^{9}$ S. Tani, Phys. Rev. 117, 252 (1960).

10M. D. Girardeau, J. Math. Phys. 16, 1901 (1975).

${ }^{11}$ M. D. Girardeau and J. D. Gilbert, Physica (Utrecht) 97A, 42 (1979).
${ }^{12}$ Gy. Csanak, H. S. Taylor, and R. Yaris, Adv. At. Mol. Phys. 7, 287 (1971).

${ }^{13}$ E. Ficocelli Varracchio, Ann. Phys. (N.Y.) 145, 131 (1983).

${ }^{14}$ S. Weinberg, Phys. Rev. 131, 440 (1963).

${ }^{15}$ M. D. Girardeau, Phys. Rev. A 26, 217 (1982).

${ }^{16}$ R. H. Bassel and E. Gerjuoy, Phys. Rev. 117, 749 (1960).

${ }^{17}$ P. J. Martin, D. M. Blankenship, T. J. Kvale, E. Redd, J. L. Peacher, and J. T. Park, Phys. Rev. A 23, 3357 (1981).

${ }^{18}$ R. Shakeshaft, Phys. Rev. A 18, 307 (1978).

${ }^{19}$ G. W. McClure, Phys. Rev. 148, 47 (1966).

${ }^{20}$ R. McCarroll, Proc. R. Soc. London 264, 547 (1961).

${ }^{21}$ J. D. Gilbert, J. Math. Phys. 18, 791 (1977).

22I. S. Gradshteyn and I. M. Ryzhik, Table of Integrals, Series and Products (Academic, New York, 1980). 